\title{
Portfolio diversification and systemic risk in interbank networks
}

\author{
Paolo Tasca ${ }^{\mathrm{a}}$, Stefano Battiston ${ }^{\mathrm{b}, *}$, Andrea Deghi $^{\mathrm{c}}$ \\ a University College London, Gower St, Bloomsbury, London, WC1E 6BT, UK \\ ${ }^{\mathrm{b}}$ University of Zurich, Rämistrasse 71, Zürich 8006, Switzerland \\ c University of Siena, Via Banchi di Sotto,55, Siena SI, Italy
}

\section{A R T I C L E I N F O}

\section{Article history:}

Received 13 August 2014

Revised 18 January 2017

Accepted 29 January 2017

Available online 9 March 2017

\section{JEL classification:}

G20

G28

Keywords:

Naive diversification

Leverage

Default probability

Financial networks

Contagion

Systemic risk

\begin{abstract}
A B S T R A C T
This paper contributes to a growing literature on the ambiguous effects of risk diversification. In our model, banks hold claims on each other's liabilities that are marked-to-market on the individual financial leverage of the obligor. The probability of systemic default is determined using a passage-problem approach in a network context and banks are able to internalize the network externalities of contagion through their holdings. Banks do not internalize the social costs to the real economy of a systemic default of the banking system. We investigate the optimal diversification strategy of banks in the face of opposite and persistent economic trends that are ex-ante unknown to banks. We find that the optimal level of risk diversification may be interior or extremal depending on banks exposure the external assets and that a tension arises whereby individual incentives favor a banking system that is over-diversified with respect to the level of diversification that is desirable in the social optimum.
\end{abstract}

(c) 2017 Published by Elsevier B.V.

\section{Introduction}

The folk wisdom of "not putting all of your eggs in one basket" has been a dominant paradigm in the financial community in recent decades. Pioneered by the works of Markowitz (1952), Tobin (1958) and Samuelson (1967), analytic tools have been developed to quantify the benefits derived from increased risk diversification. However, recent theoretical studies have begun to challenge this view by investigating the conditions under which diversification may have undesired effects (see, e.g., Battiston et al., 2012b; Ibragimov et al., 2011; Wagner, 2011; Stiglitz, 2010; Brock et al., 2009; Wagner, 2010; Goldstein and Pauzner, 2004). These works have found various types of mechanisms leading to the result that full diversification may not be optimal. For instance, Battiston et al. (2012b) assume an amplification mechanism in the dynamics of the financial robustness of banks; Stiglitz (2010) assumes that the default of one actor implies the default of all counterparties.

Our paper is closely related to Wagner (2010). They study how the trade-off between diversification and the diversity of portfolio held by different banks may lead to full diversification being suboptimal from society's perspective. The underlying assumption of their model is that in the case of a single default, the insolvent bank can sell its assets to the solvent bank and avoid physical (and more costly) liquidation. Since liquidation costs are not internalized by the banks, payoff maximizing and

\footnotetext{
* Corresponding author.

E-mail addresses: P.Tasca@ucl.ac.uk (P. Tasca), stefano.battiston@uzh.ch (S. Battiston), andrea.deghi@unisi.it (A. Deghi).
} 
social optimal levels of diversification not necessarily coincide. This implies the existence of negative externalities among the banks, whereby by increasing a bank's diversification level it also increases the possibility of costly liquidation of assets by the other, therefore augmenting the joint failures of systemic crises.

Differently from Wagner and from the aforementioned literature, this paper sheds light on a new mechanism under which a tension may arise between individual risk diversification and systemic risk. We demystify the effects of diversification in a complex context, where banks not only hold overlapping portfolios but also hold claims on each other liabilities. In this setting, a bank's payoff not only depends on the bank own financial condition, but also on the financial conditions of the other banks to which it is interconnected. We model the default of a bank as a problem of first passage time in a network-based stochastic diffusion process where the valuation of a bank liabilities relies also on the liabilities of the other banks in its network. We can show that the ambiguous role of diversification on systemic risk is much more complex and pronounced than in the setting proposed by Wagner and it involves an amplification of losses along the chain of lending relations, which - depending on the interbank network configuration - may lead the optimal degree of diversification not to be internal, as in Wagner, but to be at the corner: either minimum or maximum.

In particular, this paper assumes an arbitrage-free market where price returns are normally distributed and uncorrelated. Moreover, there are negative externalities arising from the fact that banks interbank assets are negatively affected when a counterparty's leverage increases. Banks internalize these externalities since their utility function is computed using a default probability model that accounts for the contagion from counterparties. However, the market may follow positive or negative trends that are ex-ante unpredictable and persist over a certain period of time. This incomplete information framework leads to a problem of portfolio diversification under uncertainty. In fact, portfolio returns display a bimodal distribution resulting from the combination of two opposite trends weighted by the probability of being either in a bad or in a good state of the world. We find that optimal diversification can be interior. This result holds both at the individual and at the social welfare level. Moreover, we find that individual incentives favor a financial system that is over-diversified with respect to what is socially efficient.

More in detail, we consider a banking system composed of leveraged and risk-averse financial institutions (hereafter, "banks") that invest in two asset classes. The first class consists of debts issued by other banks in the network (hereafter, "interbank claims"). The value of these securities depends, in turn, on the leverage of the issuers. The second class represents risky assets that are external to the financial network and may include, e.g., mortgages on real estate, loans to firms and households and other real economy-related activities (hereafter, "external assets"). The underlying economic cycle is the primary source of external asset price fluctuations, but it is unknown ex ante to the banks and, with a certain probability $p$, it may be positive (hereafter, "uptrend") or negative (hereafter, "downtrend"). In this paper, we focus on the effect of varying levels of diversification across external assets, while the interbank network is considered as given.

As a general property, diversification of idiosyncratic risks lowers the volatility of a bank's portfolio of external assets and increases the likelihood of the portfolio to follow the economic trend underlying the price movements. Therefore, if the future economic trend is unknown and banks cannot divest for a certain period, risk diversification is beneficial when the economic trend happens to be positive, because it reduces the downside risk. In contrast, risk diversification is detrimental when the economic trend happens to be negative because it reduces the upside potential. As a result, the intuition would suggest that the optimal level of risk diversification is always interior and depends on the probability of the market trend to be positive or negative. However, this intuition arises from the logical fallacy that maximizing a convex combination of functions is equivalent to take the convex combination of the maxima, which is not correct in general.

As a first result, we show that optimal risk diversification is interior under certain conditions, but is not interior in general. In particular, it can be optimal for individual banks to pursue a full diversification strategy even when the downtrend is almost as probable as the uptrend. Because full diversification implies that all banks are exposed to the same shocks, the probability of a systemic default conditional to an individual default tends to one. This fact leads to our second result: in a wide range of parameters there exists a tension between the individual bank's incentive to fully diversify and social optimum due to social costs associated to simultaneous defaults. Interestingly, the tension exists both when the optimal is interior and when it is an extreme point of the feasible range of $m$.

The network of interbank claims exposes banks to shocks on external assets held by their counterparties. Therefore, the network amplifies the effect of the negative trend, when it occurs, and the impact of shocks when banks have largely overlapping portfolios due to extensive diversification on external assets. ${ }^{1}$

\subsection{Related work}

One of the novelties of our work is the fact that the result about interior optimal diversification holds even in the absence of asymmetric information, behavioral biases or transaction costs and taxes. Moreover, we do not need to impose ad hoc asset price distributions as in the literature on diversification pitfalls in portfolios with fat-tailed distributions (to name a few, Zhou, 2010; Ibragimov et al., 2011; Mainik and Embrechts, 2012).

In our model, because external assets carry idiosyncratic risks, banks have an incentive to diversify across them. In this respect, similar to Bird and Tippett (1986), Elton and Gruber (1977), Evans and Archer (1968), Johnson and Shannon (1974),

\footnotetext{
1 Tasca (2013) proposed a mathematical relation between portfolio diversification and the level of overlapping (and correlation) between two portfolios.
} 
Statman (1987), we measure how the benefit of diversification vary as the external assets in an equally weighted portfolio is increased. This benchmark is the so-called $1 / n$ or naive rule. However, because banks are debt financed, we depart from the methods of those previous studies by modeling risk not in terms of a portfolio's standard deviation but in terms of the default probability. Indeed, in that literature the relationship between default probability and portfolio size has not been investigated in depth.

In order to investigate the notion of default probability in a system context we develop a framework in which banks are connected in a network of liabilities, similarly to the stream of works pioneered by Eisenberg and Noe (2001). However, that literature considers only the liquidation value of corporate debts at the time of default. In particular, in the works based on the notion of "clearing payment vector" (e.g., Cifuentes et al., 2005; Elsinger et al., 2006), the value of interbank claims depends on the solvency of the counterparties at the maturity of the contracts and it is determined as the fixed point of a so-called "fictitious sequential default" algorithm. Starting from a given exogenous shock on one or more banks, one can measure ex-post the impact of the shock in the system and investigate, for instance, which structure are more resilient to systemic risk (Battiston et al., 2012a; Roukny et al., 2013).

Our objective instead is to derive the default probability of individual banks, in a system context, that can be computed by market players ex-ante, i.e. before the shocks are realized and before the maturity of the claims. A related question was addressed in (Shin, 2008) where one assumes that asset values are random variables that move altogether according to a same scaling factor. The expected value of the assets is plugged into the Eisenberg-Noe fixed point algorithm yielding an estimate of the values of the liabilities before the observation of the shocks. However, the latter approach does not apply if assets are independent random variables and, more importantly, it does not address the issue of how the default probability of the various banks are related.

Strictly speaking, default means that the bank is not able to meet its obligations at the time of their maturity. Therefore, in principle, it does not matter whether, any time before the maturity of the liabilities, the total asset value of a firm falls beneath the book value of its debts as long as it can recover by the time of the maturity. In practice, however, it does matter a lot. This is the case, for instance, if the bank has also some short-term liabilities and short-term creditors decide to run on the bank. Indeed there is a whole literature that building on Black and Cox (1976) investigates the notion of time to default in various settings. Such notion extends the framework of Merton (1974) by allowing defaults to occur at any random time before the maturity of the debt, as soon as the firm assets value falls to some prescribed lower threshold.

Drawing inspiration from these approaches, in this paper we model the evolution over time of banks assets as stochastic processes where, at the same time, the value of interbank claims is a function of the financial leverage of the counterparties as reflected by the credit-liability network. Although from a mathematical point of view, the framework requires to deploy the machinery of continuous stochastic processes, this work offers a valuable way to compute the default probability in system context under mild assumptions. The default probability can be written in analytical form in simple cases and it can be computed numerically in more complicated cases. An underlying assumption in the model is to consider the credit spread of counterparties as an increasing function of their leverage, i.e. the higher the leverage the higher the credit spread. As a benchmark, in this paper we assume that such a dependence is linear.

In general, the framework developed here allows to investigate how the probability of defaults depends on certain characteristics of the network such as the number of interbank contracts and the number of external assets. In this paper, we focus on the diversification level across external assets and we look at the limit in which analytical results can be obtained. The assumption we make is that the interbank market is relatively tightly knit and banks are sufficiently homogeneous in balance sheet composition and investment strategies. Indeed, it has been argued that the financial sector has undergone increasing levels of homogeneity, Haldane (2009). Moreover, empirical evidence shows that bank networks feature a coreperiphery structure with a core of big and densely connected banks and a periphery of smaller banks. Thus, our hypothesis of homogeneity applies to the banks in such a core (see, e.g., Elsinger et al., 2006; Iori et al., 2006; Battiston et al., 2012c; Fricke and Lux, 2015).

The paper is organized as follows. In Section 2, we introduce the model. Section 3 adopts a marginal benefit analysis by formalizing the single bank utility maximization problem with respect to the number of external assets in the portfolio. In Section 4 we formulate the utility maximization problem in the "social optimum" and compare to the utility maximization problem of individual banks. Section 5 concludes the paper and considers some policy implications.

\section{Model}

Let time be indexed by $t \in[0, \infty]$ in a system of $N$ risk-averse leveraged banks with mean-variance utility function. To ensure simplicity in notation, we omit the time subscripts whenever there is no confusion. For the bank $i \in\{1, \ldots, N\}$, the balance sheet identity conceives the equilibrium between the asset and liability sides as follows:

$$
a_{i}=l_{i}+e_{i}, \quad \forall t \geq 0
$$

where $\mathbf{a}:=\left(a_{1}, \ldots, a_{N}\right)^{\mathrm{T}}$ is the column vector of bank assets at market value. $\mathbf{1}:=\left(l_{1}, \ldots, l_{N}\right)^{\mathrm{T}}$ is the column vector of bank debts at book face value. There is an homogeneous class of debt with maturity $T$ and zero coupons, i.e., defaultable zerocoupon bonds. $\mathbf{e}:=\left(e_{1}, \ldots, e_{N}\right)^{\mathrm{T}}$ is the column vector of equity values. Notably, the market for investment opportunities is complete and composed of two asset classes that are perfectly divisible and traded continuously: (i) $N$ interbank claims, and (ii) $M$ external assets related to the real side of the economy. There are no transaction costs or taxes. However, there are 
borrowing and short-selling restrictions. Each bank selects a portfolio composed of $n \leq N-1$ interbank claims and $m \leq M$ external assets. Then, the asset side in Eq. (1) can be decomposed $a^{2}$ :

$$
a_{i}:=\sum_{j} z_{i j} v_{j}+\sum_{k} w_{i k} \hat{\eta}_{k}
$$

In the above equation $\mathbf{Z}:=\left[z_{i j}\right]_{N \times M}$ is the $N \times M$ matrix of external investments in which each entry $z_{i j} \geq 0$ is the number of units of external asset $j$ at price $v_{j}$ held by bank $i$. $\mathbf{W}:=\left[w_{i k}\right]_{N \times N}$ is the $N \times N$ (right) stochastic matrix, i.e. the coefficient in the $(i, k)$ cell of $\mathrm{W}, w_{i k} \in[0,1]$ indicates the fraction of borrowing by $i$ from $k$. We suppose that interbank claims, denoted by $\hat{\eta}_{k}$, are marked-to-market and are priced according to the discounted value of future payoffs at maturity:

$$
\hat{\eta}_{i}=\frac{\eta_{i}}{\left(1+r_{i}\right)^{T-t}}
$$

where $\eta_{i}$ denotes the book value of bank $i$ 's obligations towards other banks in the network, i.e. the value of the payment at maturity that $i$ promised to its bond holders. $r_{i}$ is the rate of return on $(T-t)$-years maturity obligations. $c_{i}=r_{i}-r_{f}$ is the credit spread (premium), over the risk free rate $r_{f}$, paid by $i$ to the bond holders.

In a stylized form, each bank's balance-sheet is as follows:

\section{Bank-i balance-sheet}

\begin{tabular}{ll}
\hline Assets & Liabilities \\
\hline$\sum_{k} w_{i k} \hat{\eta}_{k}$ & $l_{i}$ \\
$\sum_{j} z_{i j} v_{j}$ & $e_{i}$ \\
\hline
\end{tabular}

\subsection{Leverage and default event}

Our approach to define the default in the same spirit of Black and Cox (1976), who extends Merton (1974) by allowing for a premature default when the asset value of the firm falls beneath the book value of its debt. From a technical point of view, what matters is the debt-to-asset ratio:

$$
\phi_{i}:=\frac{l_{i}}{a_{i}}
$$

$$
\text { with natural bound }[\varepsilon, 1] \text { where } \begin{cases}1 & \text { default boundary } \\ \varepsilon \rightarrow 0^{+} & \text {safe boundary. }\end{cases}
$$

Definition of default event:. The probability of the default event is the probability that $\phi_{i}$, initially at an arbitrary level $\phi_{i}(0)$ $\in(\varepsilon, 1)$, exits for the first time through the default boundary 1 , after time $t>0$. More precisely, we use the concept of first exit time, $\tau$, through a particular end of the interval $(\varepsilon, 1) .^{3}$ Namely,

$$
\tau:=\inf \left\{t \geq 0 \mid \mathbb{1}_{\phi_{i}(t) \leq \varepsilon}+\mathbb{1}_{\phi_{i}(t) \geq 1} \geq 1\right\} .
$$

If the default event is defined as $\left(\right.$ default $\left._{i}\right):=\left\{\phi_{i}(\tau) \geq 1\right\}$, then the default probability is the probability of this event:

$$
\mathbb{P}\left(\text { default }_{i}\right)=\mathbb{P}\left(\phi_{i}(\tau) \geq 1\right) \text {. }
$$

With a slight abuse of notation we rewrite $\mathbb{P}\left(\right.$ default $\left._{i}\right)$ as:

$$
\mathbb{P}\left(\text { de fault }_{i}\right)=\mathbb{P}\left(\phi_{i} \geq 1\right) \text {. }
$$

Leverage in system context:. Combining together Eqs. (2)-(4), we obtain:

$$
\phi_{i}=l_{i} /\left(\sum_{j} z_{i j} v_{j}+\sum_{k} w_{i k}\left(\frac{\eta_{k}}{\left(1+r_{k}\right)^{(T-t)}}\right)\right) .
$$

In general terms, the credit spread of bank $i$ depends on several factors such as the firm's leverage, the volatility of the underlying assets or the liquidity risk (see, e.g., Collin-Dufresne et al., 2001). In studying Eq. (6) in a system context, in order

\footnotetext{
2 Notice that, for the sake of simplicity, we omit the lower and upper bounds of the summations. It remains understood that, in the summation for external assets, the index ranges from 1 to $M$, and that, in the summation for banks, the index ranges from 1 to $N$ (with the condition that $w_{i i}=0$ for all $i \in\{1, \ldots, N\}$.

3 The notion of exit time is a crucial quantity in stochastic processes. Such notion has the important advantage to link the default event of the bank with structural (observable) variables of the bank. Hence, default event becomes a predictable event corresponding to the cases where the value of bank asset is too close to the value of its debt.
} 
to better isolate the explanatory power of leverage, here we assume that the credit spread depends only on leverage in a linear fashion:

$$
c_{i}=\beta \phi_{i} \text {. }
$$

In reality, the relation between credit spread and leverage can be more complicated. The rationale adopted in our paper is that the main determinant of the credit spread must be the distance to default, measured by the ratio $c_{i}$ of debt over total asset, thus the credit spread must be a function $f$ of such ratio: $c_{i}:=\beta f\left(\phi_{i}\right)$. It must be that the closer $\phi_{i}$ to one, the higher the probability of default and thus the higher the credit spread $c_{i}$. In other words, $f$ should be non decreasing. Moreover, because of the endogenous dynamics of asset prices, the function $f$ is likely to be highly non-linear. Since it is not possible anyway to derive it in closed form, in our paper, for the sake of simplicity, we take a parsimonious first order linear approximation, $c_{i}:=\beta f\left(\phi_{i}\right)$, which serves as a conceptually simple approximation of the original unknown function $f$. This assumption allows us to capture the main basic feature that we expect from a credit spread, namely to increase with the default probability. The parameter $\beta(>0)$ is the factor loading on $i$ 's leverage $\phi_{i}$ and can be understood as the responsiveness of the rate of return to the leverage. Then, by replacing Eq. (8) into (3) we have:

$$
\hat{\eta}_{i}=\frac{\eta_{i}}{\left(1+r_{f}+\beta \phi_{i}\right)}
$$

where, w.l.g. $T-t=1$. This means that banks issue 1 -year maturity obligations that are continuously rolled over. Notice that, by Eqs. (6) and (9), even in the case of a high default probability, bank debts are still priced at a positive market value. Namely, for $\phi_{i} \rightarrow 1, \hat{\eta}_{i}>0$. This means that, creditors are assumed to partially recover their credits in case of default.

Remark 1. If for $\phi_{i} \rightarrow 1$ the marked-to-market value of interbank liabilities is such that $\hat{\eta}_{i}>0$, the implicit recovery rate is:

$$
\delta=\left\{\begin{array}{l}
1 \text { incase of no default } \\
\frac{1+r_{f}}{1+r f+\beta} \quad \text { in case of default }
\end{array}\right.
$$

Proof: Appendix A.

Now, by using Eq. (9) we can rewrite Eq. (7) as:

$$
\phi_{i}=l_{i} /\left(\sum_{j} z_{i j} v_{j}+\sum_{k} w_{i k}\left(\frac{\eta_{k}}{1+r_{f}+\beta \phi_{k}}\right)\right) .
$$

Eq. (10) highlights a non-linear dependence of $\phi_{i}$ from the leverage $\phi_{k=1, \ldots, n}$ of the other banks to whom $i$ is exposed via the matrix $\mathbf{W}^{4}$.

Recent works based on the "clearing payment vector" mechanism (see, e.g., Eisenberg and Noe, 2001; Cifuentes et al., 2005) provide a "fictitious sequential default" algorithm to determine the liquidation equilibrium value of interbank claims at their maturity. In reality, however, defaults may happen before the maturity of the debts. In this respect, Eq. (9) together with Eq. (10) captures, even before the maturity of the debts, the market value of interbank claims in the building up of the distress spreading from one bank to another.

Remark 2. Bank's leverage defined as in Eq. (10) is a second order polynomial equation in $\boldsymbol{\Phi}$ :

$$
\boldsymbol{\Phi} \mathbf{I} \beta \boldsymbol{\Phi}+\boldsymbol{\Phi} \mathbf{I R}-\mathbf{L}(\mathbf{W H})^{-1} \beta \boldsymbol{\Phi}+\boldsymbol{\Phi}=\mathbf{L}(\mathbf{W H})^{-1} \mathbf{R}
$$

where $\quad \mathbf{H}:=\operatorname{diag}\left(\eta_{1}, \eta_{2}, \ldots, \eta_{M}\right) ; \quad \mathbf{I}:=\mathbf{Z V}(\mathbf{W H})^{-1} \quad$ and $\quad \boldsymbol{\Phi}:=\operatorname{diag}\left(\phi_{1}, \phi_{2}, \ldots, \phi_{N}\right) ; \quad \mathbf{L}:=\operatorname{diag}\left(l_{1}, l_{2}, \ldots, l_{N}\right)$; $\mathbf{V}:=\operatorname{diag}\left(v_{1}, v_{2}, \ldots, v_{M}\right) ; \mathbf{R}:=\operatorname{diag}(R, R, \ldots, R)$ with $R=1+r_{f} ; \mathbf{W}:=\left[w_{i k}\right]_{N \times N} ; \mathbf{Z}:=\left[z_{i j}\right]_{N \times M}$.

Proof: Appendix A.

Along this line of reasoning, one can notice that the default probability of a given bank depends on the likelihood of its leverage to hit the default boundary. This, in turn, depends on the joint probability of the other banks' leverages, to whom this banks is connected, of hitting the default boundary. In order to account for these network effects, in the next section we will provide an explicit form of default probability in system context.

\section{Benefits of diversification in external assets}

Similar to Bird and Tippett (1986), Elton and Gruber (1977), Evans and Archer (1968), Johnson and Shannon (1974), Statman (1987), in this section we measure the advantage of diversification by determining the rate at which risk reduction benefits are realized as the number $m(\leq M)$ of external assets in an equally weighted portfolio is increased. In contrast with those studies, rather than minimizing the variance of the banks' assets, we maximize their expected utility with respect to $m$. The methodology is explained in the following subsections. In 3.1, we formalize the equally weighted portfolio of external assets. 3.2 defines the systemic default event. In 3.4, we formalize the bank utility function and in 3.5 we maximize the utility function with respect to the control variable $m$.

\footnotetext{
${ }^{4}$ The obligation of each bank $i$ can be considered as an $n$-order derivative, the price of which is derived from the risk-free rate $r_{f}$ from the leverage $\phi_{i}$ of $i$. The latter, in turn, depends on the leverage $\phi_{k=1, \ldots, n}$ of the other banks obligors of $i$.
} 


\subsection{Equally weighted portfolio of external assets}

From Eq. (2), let bank i's portfolio of external assets be defined as:

$$
s_{i}:=\sum_{j} z_{i j} v_{j}
$$

To study the benefits of diversification, in isolation, we need to consider the $1 / \mathrm{m}$ (equally weighted) portfolio allocation. As the risk of a stock portfolio depends on the proportions of the individual stocks, their variances, and covariances, our assumption implies that the level of portfolio risk will decrease with the number of external assets $m$. Thus, every equally weighted and uncorrelated asset added to a portfolio will increase the level of diversification and reduce the portfolio risk. This allocation is adopted as a metric to measure the rate at which risk-reduction benefits are realized as the number of assets held in the portfolio is increased.

Recall that, we assume that the projects have the same average return and variance. In order for the balance-sheet identity to hold true at any time $t$ for the asset side, the fraction of assets allocated to each project must be kept constant over time (see eg. Maillard et al., 2010; Tasca et al., 2014). In other words, because the market price of external assets $s(t)$ can change for market reasons, the bank has to adjust the number of units of external assets in its portfolio with respect to the price changes in order to keep the level of investment in each external asset at $s_{i}(t) / n_{i}$. Consistently with the assumption of such portfolio rebalancing strategy of banks, we assume that external assets are equally weighted in banks' portfolios.

Formally, for every external asset $j \in\{1, \ldots, M\}$ and each bank $i \in\{1, \ldots, N\}$, the fraction of portfolio $s_{i}$ invested by bank $i$ in the external asset $j$ is:

$$
\frac{1}{m}=\frac{z_{i j} v_{j}}{s_{i}}
$$

The minimum conditions that allow us to apply the $1 / m$ rule, as a benchmark, without violating the mean-variance dominance criterion, is to assume the external assets are statistically indistinguishable, i.e., they have the same drift, the same variance and they are uncorrelated. ${ }^{5}$

Thus, the price of external assets is properly characterized by following time-homogeneous diffusion process: ${ }^{6}$

$$
\frac{d v_{j}}{v_{j}(t)}=\mu d t+\sigma d \tilde{B}_{j}(t), \quad j=1, \ldots, M
$$

Using the expression in Eq. (13), we arrive after some transformations at the following dynamics for the the portfolio in Eq. (12):

$$
\frac{d s_{i}}{s_{i}(t)}=\mu d t+\frac{\sigma}{\sqrt{m}} d B_{i}(t) .
$$

$B_{i}=\frac{1}{m} \sum_{j} \tilde{B}_{j}$ is an equally weighted linear combination of Brownian shocks s.t. $d B_{j} \sim N(0, d t)$.

Properties of the portfolio:

There exist two states of the world, $\theta=\{0,1\}$. This captures a situation in which the economy is either in a boom $(\theta=1)$ or in a bust $(\theta=0)$ state and is reminiscent of a stylized economic cycle. The probability that the world is in state $\theta$ is denoted as $\mathbb{P}(\{\theta\})$ with $\mathbb{P}(\{0\})=p$ and $\mathbb{P}(\{1\})=1-p$. According to the state of the world, the market of external assets is assumed to follow a given constant stochastic trend under a certain probability space $\left(\Omega_{\mu}, \mathcal{A}, \mathbb{P}\right)$. The sample space $\Omega_{\mu}=\left\{\mu^{-}, \mu^{+}\right\}$is the set of the outcomes. We use the convention:

$$
\begin{cases}\mu<0:=\mu^{-} & \text {if } \theta=0, \\ \mu \geq 0:=\mu^{+} & \text {if } \theta=1\end{cases}
$$

with $\left|\mu^{+}\right|=\left|\mu^{-}\right|$. The $\sigma$-algebra $\mathcal{A}$ is the power set of all the subsets of the sample space, $\mathcal{A}=2^{\Omega \mu}=2^{2}=$ $\left\{\left\{\mu^{-}\right\},\left\{\mu^{+}\right\},\left\{\mu^{-}, \mu^{+}\right\},\{\}\right\}$is the probability measure, $\mathbb{P}: \mathcal{A} \rightarrow[0,1]$ with $\mathbb{P}(\{\})=0, \mathbb{P}\left(\left\{\mu^{-}\right\}\right)=p$ and $\mathbb{P}\left(\left\{\mu^{+}\right\}\right)=1-p$. That is, $p$ and $(1-p)$ are the probabilities of having a downtrend and an uptrend, respectively.

To conclude, portfolio returns display a mixture distribution expressed by the convex combination of two normal distributions weighted by $p$ and $1-p$. Namely,

$$
\frac{d s_{i}}{s_{i}} \sim p N\left(\mu^{-}, \frac{\sigma}{\sqrt{m}}\right)+(1-p) N\left(\mu^{+}, \frac{\sigma}{\sqrt{m}}\right)
$$

\footnotetext{
${ }^{5}$ Notice that under those conditions, the $1 / m$ portfolio allocation is Pareto optimal. See e.g., Rothschild and Stiglitz (1971), Samuelson (1967), Windcliff and Boyle (2004).

${ }^{6}$ Where $\tilde{B}_{j}(t)$ is a standard Brownian motion defined on a complete filtered probability space $\left(\Omega ; \mathcal{F} ;\left\{\mathcal{F}_{t}\right\} ; \mathbb{P}\right)$, with $\mathcal{F}_{t}=\sigma_{y}\{\tilde{B}(s): s \leq t\}, \mu$ is the instantaneous risk-adjusted expected growth rate, $\sigma>0$ is the volatility of the growth rate and $\mathbb{E}\left(d \tilde{B}_{j}, d \tilde{B}_{y}\right):=\rho_{j y}=0$.
} 


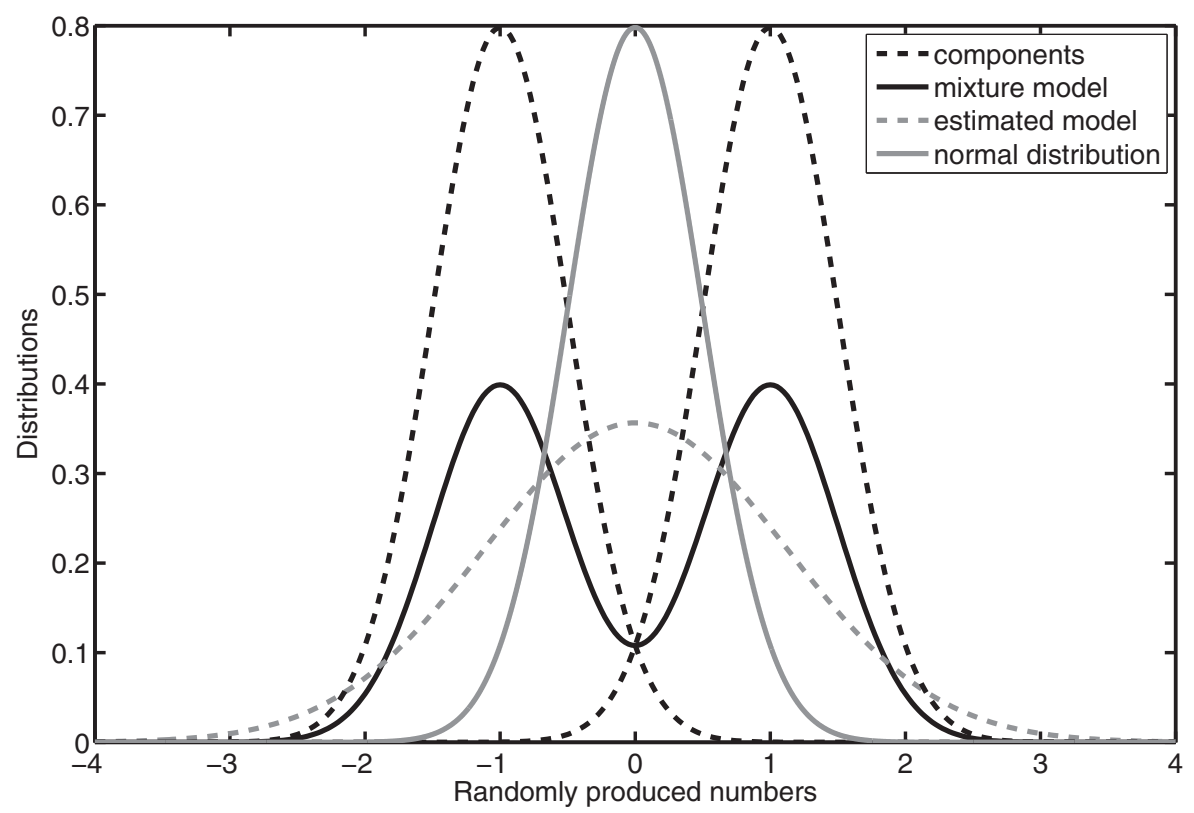

Fig. 1. Distribution of portfolio returns (mixture model). Comparison between a portfolio with returns following a bimodal distribution (black color) and a portfolio with normally distributed returns (gray colour). Parameters: $\mu=0, \mu^{-}=-1, \mu^{+}=1, \sigma=0.5, p=0.5$.

$$
\text { with }\left\{\begin{array}{l}
\mathbb{E}\left[\frac{d s_{i}}{s_{i}}\right]:=\hat{\mu}=p \mu^{+}+(1-p) \mu^{-} \\
\mathbb{E}\left[\left(\frac{d s_{i}}{s_{i}}-\hat{\mu}\right)^{2}\right]:=\hat{\sigma}^{2}=p\left[\left(\mu^{+}-\hat{\mu}\right)^{2}+\frac{\sigma^{2}}{m}\right]+(1-p)\left[\left(\mu^{-}-\hat{\mu}\right)^{2}+\frac{\sigma^{2}}{m}\right]
\end{array}\right.
$$

Fig. 1 illustrates this result by comparing two probability density functions (pdf). The first (gray color) curve represents the pdf of a portfolio with returns $X$ normally distributed, i.e., $X \sim N(0,1)$. The second (black color) curve represents the pdf of a portfolio with returns distributed as $Y 1 \sim N(1,0.5)$ with probability $p=0.5$ and as $Y 2 \sim N(-1,0.5)$ with probability $1-p=0.5$. Notice that in a world where the market displays a normal distribution, the middle part of the distribution range (the "belly") is the most likely outcome. In contrast, in a bimodal world, the belly is the least likely outcome. Moreover, the tails of the bimodal distribution are higher than those of the normal distribution. This indicates the higher probability of severe left and right side events. The distance between the two peaks depends on the difference between the means of the two normal distributions, $\left|\left(\mu^{+}\right)-\left(\mu^{-}\right)\right|$. The (possible) asymmetry between the two peaks and the skewness of the bimodal distribution depends on the difference between the probability of having an uptrend or a downtrend, $|(1-p)-p|$. As a result, portfolio diversification choice is subject to much more uncertainty in a bimodal world than in a normal one. The next sections show how the statistical properties of the bimodal distribution impact on the risk diversification effects.

\subsection{Systemic default probability}

Interbank network structure and contagion. The model described above can be used to address several questions. In this paper, we analyze the properties of the model in a specific scenario.

It has been argued that the financial sector has undergone increasing levels of homogeneity, Haldane (2009). Moreover, empirical evidence shows that bank networks feature a core-periphery structure with a dense core of fully connected banks and a periphery of small banks. Thus, an hypothesis of homogeneity is realistic at least for the banks in the core (see, e.g., Elsinger et al., 2006; Iori et al., 2006; Battiston et al., 2012c).

We therefore assume that (i) banks have homogeneous capital structure, (ii) hold similar balance sheets and portfolios, and (iii) hold claims on each other liabilities in a tightly knit interbank network.

Furthermore, we do not consider here issues related to endogenous interbank network formation, optimal interbank network structures and network efficiency. See, e.g. Gale and Kariv (2007), Castiglionesi and Navarro (2007) and the survey by Allen and Babus (2009) for discussion of these topics.

Relation between individual and systemic default. Under the assumption of homogeneity, banks have the same capital structure:

- the portfolio of external assets is similar across banks, i.e., $s_{i}=s$ for all $i \in\{1, \ldots, N\}$;

- the book value of promised payments at maturity is equal for every bank, i.e., $l_{i}=l$ for all $i \in\{1, \ldots, N\}$. 


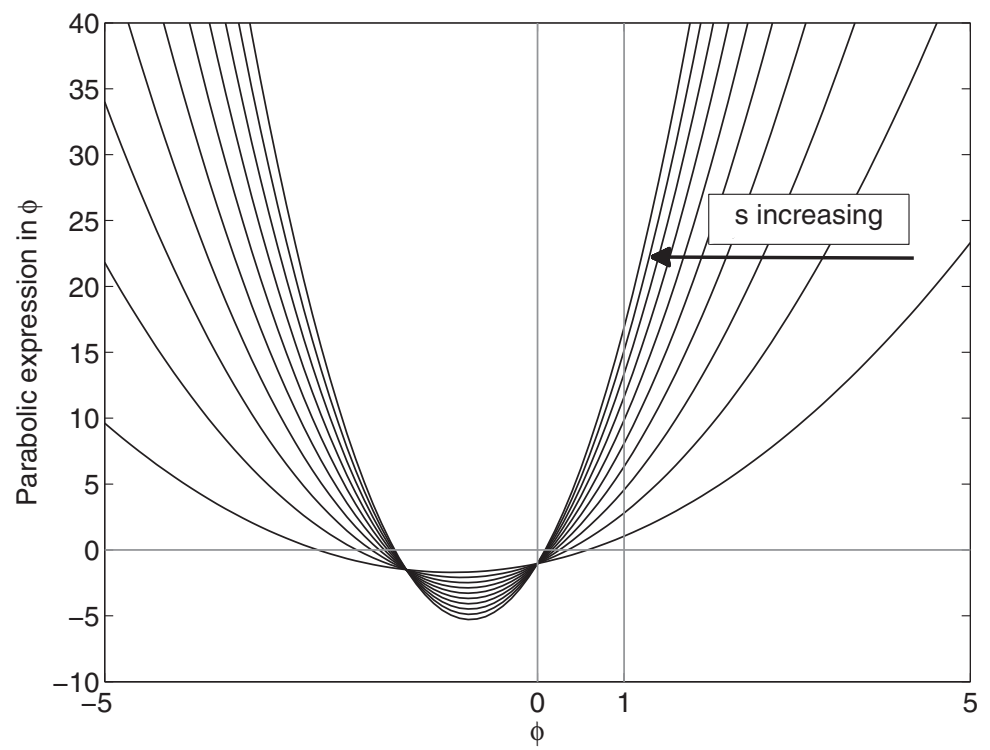

Fig. 2. Parabolic expression of the market leverage. The market leverage is the solution of a parabolic expression that implies a non linear relationship between banks' leverage. Parameters: $l=0.9, s \in\{1,2, \ldots, 10\}, r_{f}=0.03, \beta=0.5, w=1$.

The last assumption implies a symmetry between liabilities of bank $i, l_{i}$ and the face value of its interbank assets, $\eta_{k}$ in Eq. (10). Under those conditions, leverage levels among banks are equal. ${ }^{7}$ Namely, the leverage of every bank will be equal to the market leverage:

$$
\phi_{i}=\frac{1}{N} \sum_{j}^{N} \phi_{j}:=\phi,
$$

for all $i \in\{1, \ldots, N\}$. The above assumption, together with the assumption that $l_{i} \equiv \eta_{k} \equiv l$ and the results from the previous section, allow us to rewrite Eq. (10) as:

$$
\phi=l /\left(s+w \frac{l}{1+r_{f}+\beta \phi}\right) .
$$

where $w \in[0,1]$ indicates the fraction of borrowing of banks in the interbank.

Eq. (17) is a quadratic expression in $\phi$ :

$$
\phi^{2} \beta s+\phi(s R+l(1-\beta))-w l R=0, \quad R=1+r_{f} .
$$

Numerically, we find that Eq. (18) has always one positive and one negative root for any values of the parameters $(\beta>0, R$ $\geq 1, s>0, l>0$ ) in their range of variation:

$$
\left\{\begin{array}{l}
\phi_{\text {pos }}=\frac{1}{2 \beta s}\left[l(\beta-1)-R s+\left(4 \beta l R s w+(l(1-\beta)+R s)^{2}\right)^{1 / 2}\right] \\
\phi_{\text {neg }}=\frac{1}{2 \beta s}\left[l(\beta-1)-R s-\left(4 \beta l R s w+(l(1-\beta)+R s)^{2}\right)^{1 / 2}\right]
\end{array}\right.
$$

Since, by definition $\phi$ can only be positive, we exclude the negative solution. Therefore, one can always find a unique positive solution to Eq. (18):

$$
\phi:=\phi_{\text {pos }} \text {. }
$$

The parabola in Eq. (18) is depicted in Fig. 2. For different values of $s$ the roots are those points crossing zero in the interval $[\varepsilon, 1]$, which is the natural bound of the leverage, see Eq. (4).

The setting described above motivates the assumption that the leverage of the individual bank approximates the leverage of the system:

$$
\phi_{i} \simeq \phi
$$

\footnotetext{
7 The validity of such approximation increases with the level of homogeneity across banks. Because the empirical literature consistently reports the existence of densely interconnected core of banks in interbank markets, the assumption intends to capture situations like the one of 2007-2009, in which a core of banks was highly connected and heavily exposed to the same mortgage backed securities. This operation on one hand makes the variables homogeneous, but allows us, on the other hand, to explore the systemic effects arising from banks' interconnections while being parsimonious on the assumptions.
} 
for all $i \in\{1, \ldots, N\}$. Then, if we apply Eq. (6), for the continuous mapping theorem ${ }^{8}$ we obtain:

$$
\mathbb{P}\left(\text { default }_{i}\right)=\mathbb{P}\left(\phi_{i} \geq 1\right) \simeq \mathbb{P}(\text { default })=\mathbb{P}(\phi \geq 1) .
$$

for all $i \in\{1, \ldots, N\}$.

The systemic default probability, $\mathbb{P}$ (default), depends on the process governing the leverage $\phi(t)$ that, in turn, depends on the process governing the value of the portfolio of external assets $s(t)$. Because the relation between $\phi(t)$ and $s(t)$ is monotonic, there is a mapping between the passage times of the two processes and the systemic default probability can be computed more easily in terms of $s$ (see Appendix A):

$$
\begin{aligned}
& \mathbb{P}(\text { default })=\mathbb{P}(\phi \geq 1) \equiv \mathbb{P}\left(s \leq s^{-}\right) \\
& \text {with }\left\{\begin{array}{lll}
s^{+} & =\frac{l(\beta \varepsilon+R-w \varepsilon)}{\varepsilon(\beta \varepsilon+R)} & \text { safe boundary } \\
s^{-} & =\frac{l(\beta+R-w)}{\beta+R} & \text { default boundary. }
\end{array}\right.
\end{aligned}
$$

Proposition 1. Consider the default conditions in Eq. (22) and assume that external assets dynamics follows a time-homogeneous process with the same drift and same variance. If external assets are uncorrelated, $\mathbb{P}$ (default), can be expressed as

$$
\mathbb{P}(\text { default })=\left(\int_{s_{0}}^{s^{+}} d s \psi(x)\right) /\left(\int_{s^{-}}^{s^{+}} \mathrm{d} x \psi(x)\right) \text {, where } \psi(x)=\exp \left(\int_{0}^{x}-\frac{2 \hat{\mu}}{\hat{\sigma}^{2}} \mathrm{~d} s\right) .
$$

and has the following closed form solution:

$$
\mathbb{P}(\text { default })=\left(\exp \left[-\frac{2 \hat{\mu} s_{0}}{\hat{\sigma}^{2}}\right]-\exp \left[-\frac{2 \hat{\mu} s^{+}}{\hat{\sigma}^{2}}\right]\right) /\left(\exp \left[-\frac{2 \hat{\mu} s^{-}}{\hat{\sigma}^{2}}\right]-\exp \left[-\frac{2 \hat{\mu} s^{+}}{\hat{\sigma}^{2}}\right]\right) .
$$

Proof: see Appendix A.

Eq. (A.4) is the probability that $s$, initially at an arbitrary level $s(0):=s_{0} \in\left(s^{-}, s^{+}\right)$, exits through $s^{-}$before $s^{+}$after time $t>0$. This can be related to the problem of first exit time through a particular end of the interval $\left(s^{-}, s^{+}\right)$(Gardiner, 1985).

Now we need the following definition.

Definition 4. The conditional systemic default probability conditional to a given market trend is :

$$
\begin{cases}q:=\mathbb{P}\left(\text { default } \mid \mu^{-}\right) & \text {def. prob. in the case of a downtrend } \\ g:=\mathbb{P}\left(\text { default } \mid \mu^{+}\right) & \text {def. prob. in the case of an uptrend }\end{cases}
$$

From Proposition 1 and Definition 4 we can now derive the following proposition:

Proposition 2. The conditional systemic default probability, conditional to a given market trend has the following closed form solutions:

$$
\left\{\begin{array}{l}
q=\left(\exp \left[-\frac{\left(2 \mu^{-}\right) s_{0}}{\sigma^{2} m}\right]-\exp \left[-\frac{\left(2 \mu^{-}\right) s^{+}}{\sigma^{2} m}\right]\right) /\left(\exp \left[-\frac{\left(2 \mu^{-}\right) s^{-}}{\sigma^{2} / m}\right]-\exp \left[-\frac{\left(2 \mu^{-}\right) s^{+}}{\sigma^{2} / m}\right]\right), \\
g=\left(\exp \left[-\frac{\left(2 \mu^{+}\right) s_{0}}{\sigma^{2} / m}\right]-\exp \left[-\frac{\left(2 \mu^{+}\right) s^{+}}{\sigma^{2} / m}\right]\right) /\left(\exp \left[-\frac{\left(2 \mu^{+}\right) s^{-}}{\sigma^{2} / m}\right]-\exp \left[-\frac{\left(2 \mu^{+}\right) s^{+}}{\sigma^{2} / m}\right]\right) .
\end{array}\right.
$$

Proof: Appendix A.

In such setting, the first passage time of this process must account for the new boundary. A bank could in principle reach at time $t^{i}>0$ the safe boundary $\varepsilon$ and then only later at time $t^{i i}>t^{i}>0$ reach the default boundary at 1 . Notice, however, that the probability of such an event is very low and this may occur only in a scholastic example when: (1) the variance $\sigma$ is much larger than the trend $\mu$; (2) the two barriers $s^{+}$and $s^{-}$are very close to each other; and (3) the initial value of external assets $s_{0}$ is very close to the boundaries. ${ }^{9}$

Diversification effects on default probability. The analysis of $q$ and $g$ reveals that, in an idealized world without transaction costs and infinite population size of external assets (i.e., $M \rightarrow \infty$ ), at increasing levels of risk diversification (i.e., $m \rightarrow M$ ), the default probability exhibits a bifurcated behavior when trends are persistent (i.e., approximately constant during a given period $\Delta t$ ) See Fig. 3. We can formalize the result in the following proposition.

Proposition 3. Consider a debt-financed portfolio subject to a given default threshold as in the model described so far. Then, the default probability decreases with risk diversification in case of asset prices uptrend and increases with diversification in case of asset prices downtrend.

Proof: see Appendix A.

The intuition behind the polarization of the probability to "survive" and the probability to "fail" is simple, but its implications are profound. In brief, the diversification of idiosyncratic risks reduces the volatility of the portfolio. The lower volatility increases the likelihood of the portfolio to follow an underlying economic trend. Therefore:

\footnotetext{
${ }^{8}$ In a nutshell, the theorem states that continuous functions preserve limits even if their arguments are sequences of random variables. See Billingsley (1968).

${ }^{9}$ See also Appendix B for a numerical validation of the approximation.
} 


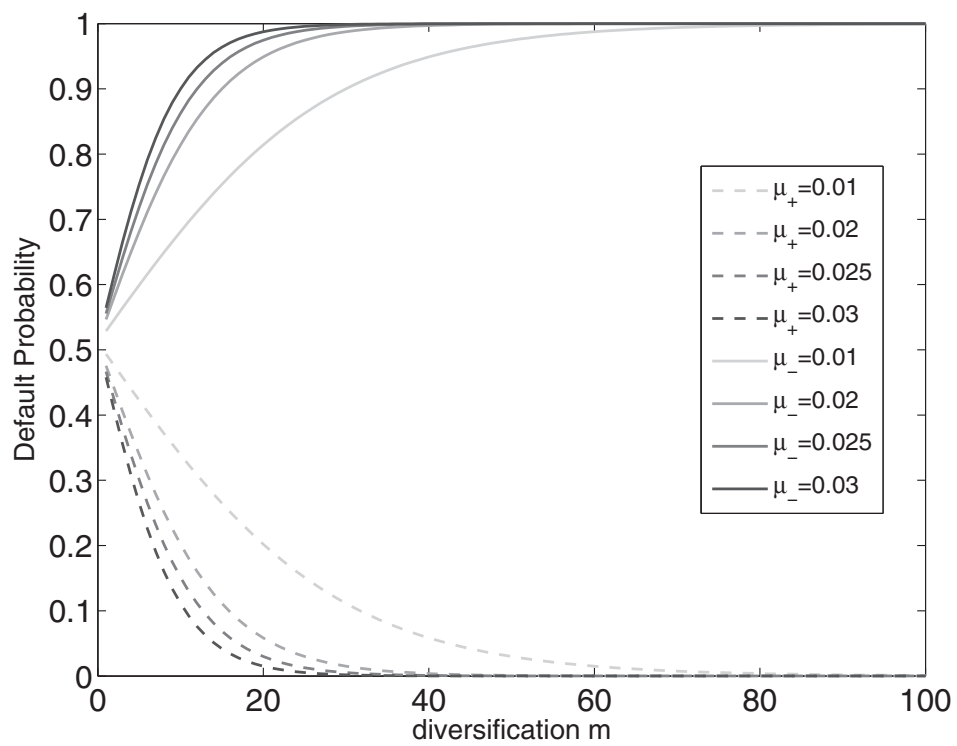

Fig. 3. Default probability for different levels of risk diversification. Default probability $q$ given a downtrend (solid lines). Default probability $g$ given an uptrend (dashed lines). $q$ increases with diversification. Instead, $g$ decreases with diversification. The elasticity of $q$ and $g$ with respect to $m$ depends on the magnitude of the trend. $\left|\mu_{+}\right|=\left|\mu_{-}\right| \in\{0.01,0.02,0.025,0.03\}, l=0.4, s_{0}=\left(s^{+}-s^{-}\right) / 2, \sigma^{2}=0.5, r_{f}=0.01, \epsilon=0.1, \beta=0.2, w=1$.

a

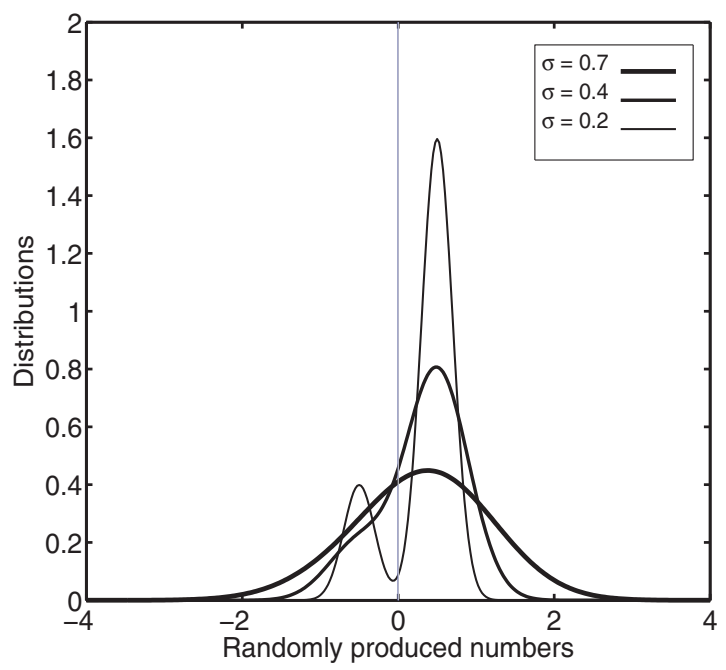

b

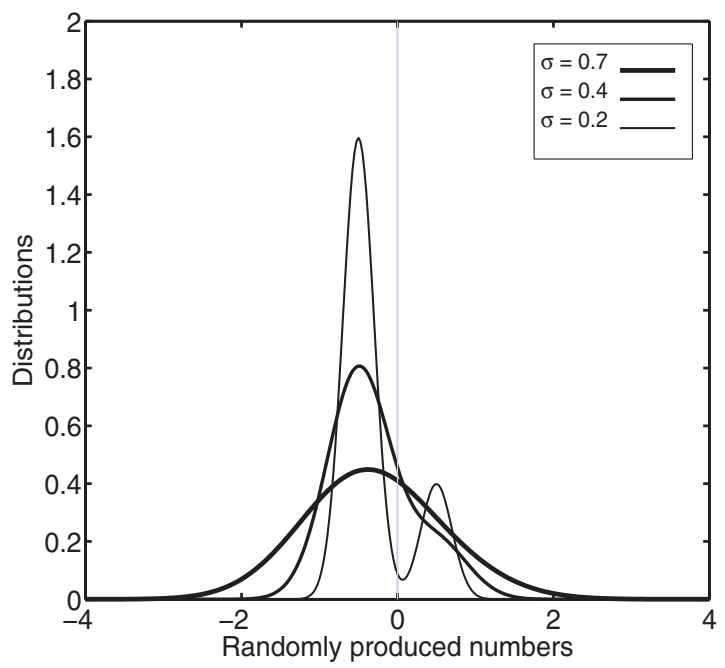

Fig. 4. Distribution of portfolio returns for different levels of volatility and different prob. of downtrend. Comparison between the probability density functions of three portfolios with returns following a bimodal distribution with the same expected value but decreasing volatility. Thick black curve ( $\sigma=$ $0.7)$, medium black curve $(\sigma=0.4)$, thin black curve $(\sigma=0.2)$. (a) Each curve represents the pdf of a portfolio with returns distributed as $Y 1 \sim N(-0.5, \sigma)$ with probability $p=0.2$ and as $Y 2 \sim N(0.5, \sigma)$ with probability $1-p=0.8$. (b) Each curve represents the pdf of a portfolio with returns distributed as $Y 1$ $\sim N(0.5, \sigma)$ with probability $p=0.8$ and as $Y 2 \sim N(0.5, \sigma)$ with probability $1-p=0.2$.

- in uptrend periods, diversification is beneficial because it reduces the downside risk and highlights the positive trend; thus, the default probability decreases;

- in downtrend periods, diversification is detrimental because it reduces the upside potential and highlights the negative trend; therefore, the default probability increases.

Fig. 4 explains this intuition by showing how the pdf of portfolio returns is influenced by the downtrend probability and by the level of diversification. As one may observe, for increasing diversification, viz., lower volatility, the pdf changes shape by moving from the thick black curve $(\sigma=0.7)$, to the medium black curve $(\sigma=0.4)$, and finally to the thin black curve $(\sigma=0.2)$. In Fig. 4a the probability of a positive trend is greater than the probability of a negative trend, $p=0.2$. 
a

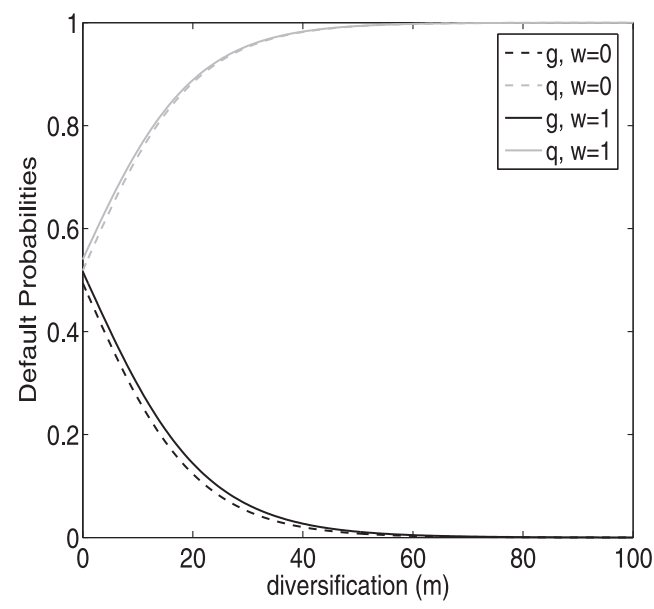

b

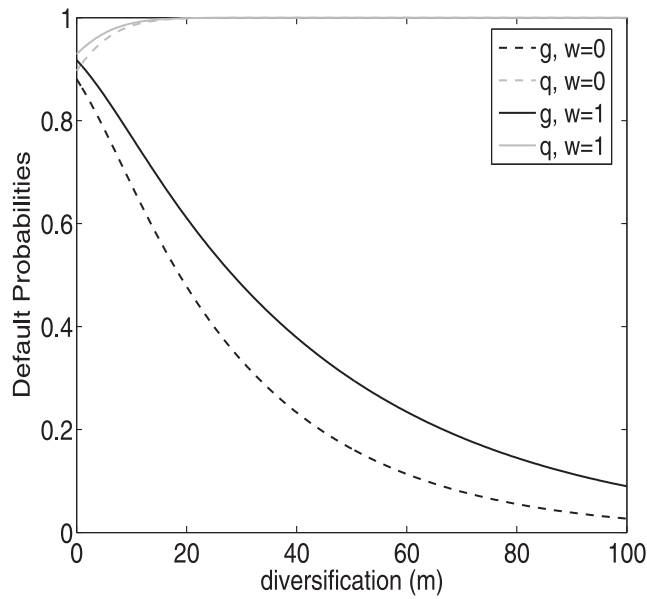

Fig. 5. Probabilities of default with and without interbank. Comparison between def. probabilities $g$ and $q$ with interbank exposures (bold lines) and without interbank exposures among banks (dashed lines). Eliminating interbank spillovers from the setting decreases in both scenarios the probabilities of default $q$ and $g$. We test for different values of $s_{0}$. Parameters: (a) $l=0.9, s_{0}=4, p=0.4, \beta=0.5, r_{f}=.001, \epsilon=0.1, w \in\{0,1\} ;(\mathbf{b}) l=1.5, s_{0}=1.5$, $p=0.4, \beta=0.5, r_{f}=.001, \epsilon=0.1, w \in\{0,1\}$.

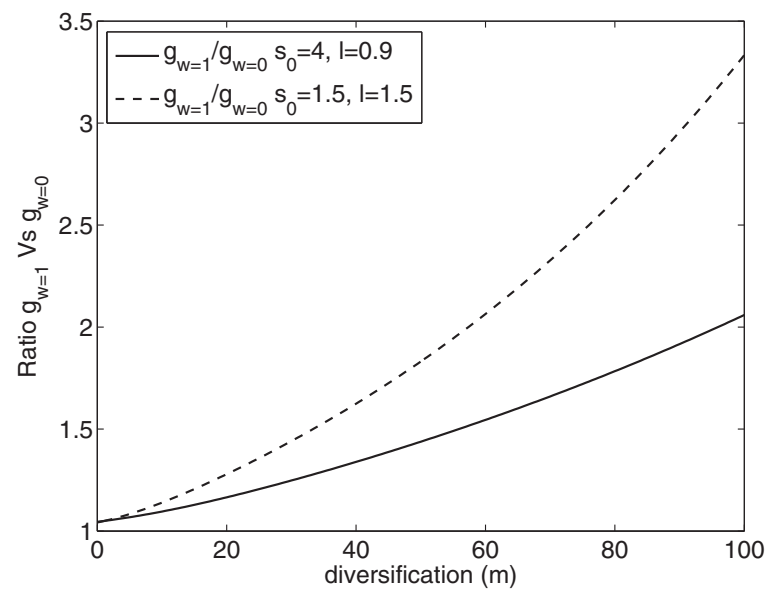

Fig. 6. Ratio between probabilities of default $g$ calculated with and without interbank. Ratio, $\frac{g_{w=1}}{g_{w-1}}$, between the default probability $g$ (conditional to uptrend) in the case with and without interbank exposures. The following parameter sets are considered: solid lines: $l=0.9, s_{0}=4, p=0.4, \beta=0.5$, $r_{f}=.001, \epsilon=0.1, w \in\{0,1\}$; dashed line: $l=1.5, s_{0}=1.5, p=0.4, \beta=0.5, r_{f}=.001, \epsilon=0.1, w \in\{0,1\}$.

Therefore, diversification is desirable because it reduces the volatility and, by doing so, it shifts to the right the density of the distribution of portfolio returns. Instead, In Fig. $4 \mathrm{~b}$ the probability of a negative trend is greater than the probability of a positive trend, $p=0.8$. In this case, diversification is undesirable because, by reducing the volatility, it shifts to the left the density of the distribution.

\subsection{The effect of network on default probability}

In order to illustrate the effect of the interbank network, we compare the case in which banks are connected in an interbank market $(w=1)$ with the case in which banks are not exposed to the interbank market $(w=0)$. However, in order to make the balance sheets comparable in the two cases in terms of leverage, we replace the interbank claims with other independent assets of equivalent face value. These latter assets are independent from the external assets and are constant in value. In these cases, we compute the closed form solutions for the default probabilities $q$ and $g$ in the two scenarios.

In Fig. 5, we plot the probabilities $g$ and $q$ for increasing levels of diversification. Dashed lines refer to the case of isolated banks, while solid lines indicate the case of interbank exposures. As we can see, the default probability with interbank exposures is always higher than the corresponding default probability in the case of isolated banks. This holds true both for the scenario of uptrend and downtrend. Moreover, in Fig. 6 we plot the ratio between the default probability conditional to the downtrend $g, \frac{g_{w=1}}{g_{w=0}}$, in the case with and without interbank exposures, for different portfolio leverage values. In the 
two examples shown, the ratio between the two curves increases either because of a higher level of diversification $m$ (along $x$-axis) or because of the balance sheet structure (e.g. the values of $s_{0}$ and $l$, see bold line vs dashed line in Fig. 6). On one hand, with higher level of leverage $\phi$ (dashed line), the average effect of the network externalities on banks becomes stronger because, whenever an agent suffers a relatively larger shock, the impact is reinforced by the network structure and the initial default of one agent causes a big loss to the counterparties that are already fragile $(\phi \approx 1)$; on the other hand, since a greater level of diversification implies a lower probability of default in case of downtrend, the results indicate that the impact of network effect is larger for a lower level default probability of the bank.

The reason behind these results is the fact that increases in leverage of the counterparties $k$ of the bank $i$ cause an increase in the leverage of bank $i$ through the market value of the interbank claims. This effect is proportional to the exposure of the bank to the interbank market. This is stated in the following proposition.

Proposition 4. Assume homogeneity in the banks' capital structure and the presence of a network of interbank exposures among banks. For a given default threshold, ceteris paribus, the leverage of an individual bank, measured in terms of its leverage $\phi$, increases non-linearly with the level of its borrowers leverage and proportionally to the level of its interbank exposures.

Proof: Appendix A.

We conclude by saying that, for a given change in counterparties' leverage $\phi_{k}$, an increment in both the interbank network density $(w \rightarrow 1)$ and in the level of diversification $(m \rightarrow M)$ amplifies the variations of individual banks' leverage and their default probability.

\subsection{Bank utility function}

In this section, we formalize the bank utility maximization problem with respect to the number $m$ of external assets held in the equally weighted portfolio described in Section 3.1.

The bank's payoff from investing in external assets is a random variable $\Pi_{m}$ that depends on the number $m$ of external assets in portfolio and on their values. It takes the value $\pi$ in the set $\Omega_{\pi}=\left[\pi^{-}, \ldots, \pi^{+}\right]$, where:

$$
\begin{cases}\pi^{-}:=s^{-}-s_{0} & \text { max attainable loss, } \\ \pi^{+}:=s^{+}-s_{0} & \text { max attainable profit. }\end{cases}
$$

More specifically, given $m$ mutually exclusive choices (i.e., the bank portfolio can be composed of $1,2, \ldots$ external assets) and their corresponding random return $\Pi_{1}, \Pi_{2}, \ldots$, with distribution function $F_{1}(\pi), F_{2}(\pi), \ldots$, preferences that satisfy the von Neumann-Morgenstern axioms imply the existence of a measurable, continuous utility function $U(\pi)$ such that $\Pi_{1}$ is preferred to $\Pi_{2}$ if and only if $\mathbb{E} U\left(\Pi_{1}\right)>\mathbb{E} U\left(\Pi_{2}\right)$. We assume that banks are mean-variance (MV) decision makers, such that the utility function $\mathbb{E} U\left(\Pi_{m}\right)$ may be written as a smooth function $V\left(\mathbb{E}\left(\Pi_{m}\right), \sigma^{2}\left(\Pi_{m}\right)\right)^{10}$ of the mean $\mathbb{E}\left(\Pi_{m}\right)$ and the variance $\sigma^{2}\left(\Pi_{m}\right)$ of $\Pi_{m}$ or

$$
V\left(\mathbb{E}\left(\Pi_{m}\right), \sigma^{2}\left(\Pi_{m}\right)\right):=\mathbb{E} U\left(\Pi_{m}\right)=\mathbb{E}\left(\Pi_{m}\right)-\left(\lambda \sigma^{2}\left(\Pi_{m}\right)\right) / 2
$$

such that $\Pi_{1}$ is preferred to $\Pi_{2}$ if and only if ${ }^{11}$

$$
V\left(\mathbb{E}\left(\Pi_{1}\right), \sigma^{2}\left(\Pi_{1}\right)\right)>V\left(\mathbb{E}\left(\Pi_{2}\right), \sigma^{2}\left(\Pi_{2}\right)\right) .
$$

Then, the maximization problem is as follows:

$$
\begin{aligned}
& \max _{m} \mathbb{E} U\left(\Pi_{m}\right)=\mathbb{E}\left(\Pi_{m}\right)-\frac{\lambda \sigma^{2}\left(\Pi_{m}\right)}{2} \\
& \text { s.t.: }\left\{\begin{array}{l}
1 \leq m \leq M \\
l>0 \\
s^{-}<s_{0}<s^{+} \\
p \mu_{s}^{-}+(1-p) \mu_{s}^{+}>0
\end{array}\right. \\
& \text { with }\left\{\begin{array}{l}
\mathbb{E}\left(\Pi_{m}\right)=p\left[q \pi^{-}+(1-q) \pi^{+}\right]+(1-p)\left[g \pi^{-}+(1-g) \pi^{+}\right] \\
\sigma^{2}\left(\Pi_{m}\right)=p\left[q\left(\pi^{-}-\mathbb{E}\left(\Pi_{m}\right)\right)^{2}+(1-q)\left(\pi^{+}-\mathbb{E}\left(\Pi_{m}\right)\right)^{2}\right] \\
+(1-p)\left[g\left(\pi^{-}-\mathbb{E}\left(\Pi_{m}\right)\right)^{2}+(1-g)\left(\pi^{+}-\mathbb{E}\left(\Pi_{m}\right)\right)^{2}\right] .
\end{array}\right.
\end{aligned}
$$

Notice that Eq. (24) is a static non-linear optimization problem with respect to $m$, with inequality constraints. ${ }^{12}$

\footnotetext{
${ }^{10}$ To describe $V$ as smooth, it simply means that $V$ is a twice differentiable function of the parameters $\mathbb{E}\left(\Pi_{m}\right)$ and $\sigma^{2}\left(\Pi_{m}\right)$.

11 Only the first two moments are relevant for the decision maker; thus, the expected utility can be written as a function in terms of the expected return (increasing) and the variance (decreasing) only, with $\partial V\left(\mathbb{E}\left(\Pi_{m}\right), \sigma^{2}\left(\Pi_{m}\right)\right) / \partial \mathbb{E}\left(\Pi_{m}\right)>0$ and $\partial V\left(\mathbb{E}\left(\Pi_{m}\right), \sigma^{2}\left(\Pi_{m}\right)\right) / \partial \sigma^{2}\left(\Pi_{m}\right)<0$.

12 Although the realized state of the world will be either an uptrend or a downtrend, banks implement their portfolio diversification strategy before the realization of the trend. Under this condition of uncertainty, from the perspective of portfolio selection problem, returns display a mixture distribution (expressed by the convex combination of two normal distributions weighted by $p$ and $1-p$ ) as shown in Eq. (16). Coherently, also the expected utility in Eq. (24) follows a similar mixture property with expected profit and variance expressed by the convex combination of two alternative payoffs weighted by $p$ and $1-p$.
} 
The first constraint means that $m$ can take only positive values between 1 and $M$. The second constraint requires banks to be debt-financed. The third constraint requires that banks are not yet in default when implementing their asset allocation, i.e., the initial portfolio value must lie between the lower default boundary and the upper safe boundary. The last constraint represents the "economic growth condition". That is, the expected economic trend of the real economy-related assets has to be positive. Since by definition, $\left|\mu_{s}^{-}\right|=\left|\mu_{s}^{+}\right|=\mu_{s}$, this condition is equivalent to impose: (1) a lower bound on the trend, namely $\mu_{s}>0$, and (2) an upper bound to the downtrend probability, namely $p \in \Omega_{p}:=\left[0, \frac{1}{2}\right)$. Then, given the above constraints, at time $t$ banks randomly select (and fix) the number $m$ of external assets to hold in their portfolio in order to minimize their default probability. This, in turn, maximizes their expected utility.

Finally, in the next section we will show that the expected utility in Eq. (24) is lower when banks hold external assets with higher initial values, $s_{0}$. This counter-intuitive property is explained by the non-linear payoff function formalized at the beginning of this section. A higher initial price $s_{0}$ increases maximal losses and decreases maximal profits. For example, if the maximum (minimum) attainable value by the external assets is $100(0)$ then, when $s_{0}=90$ the maximum profit (loss) is 10 (90). Instead, if the $s_{0}=10$, the maximum profit (loss) is 90 (10). This effect combines non-linearly into Eq. (24) and leads to a higher (lower) bank expected utility for lower (higher) levels of $s_{0}$. This mechanism resembles profit/loss dynamics from investments in a boom-bust cycle. Banks buy assets at high (low) price in a boom (bust) period. Correspondingly, in absolute terms, their maximum attainable profit is lower (higher) and their maximum attainable loss is higher (lower).

\subsection{Solution of the bank max problem}

Notice that, the exposure of banks to external assets is both direct through their own portfolio and indirect, through their interbank claims on counterparties who are also exposed to similar portfolios of external assets. In the following, banks compute the optimal level of diversification taking this indirect effect into account. In other words, because the expression for the probability of systemic default takes into account the effect of contagion through the interbank market, banks internalize the negative externalities of contagion in their utility function.

Banks face the ex-ante uncertainty on which economic trend will occur and they know that they will not be able to divest from their external assets. As shown in the previous sections, maximal risk diversification minimizes the default probability if the economic trend happens to be positive, because it reduces the downside risk. In contrast, a minimal risk diversification minimizes the default probability when the economic trend happens to be negative. The intuition could then suggest that the optimal level of risk diversification is always interior and depends on the probability of the market trend to be positive or negative. However, this intuition suffers from the logical fallacy that maximizing a convex combination of functions is equivalent to take the convex combination of the maxima, which is not correct in general.

We show that optimal risk diversification is interior under certain conditions, but is not interior in general. In particular, we find that:

- banks with small enough share of asset invested in the external assets $\left(s_{0}<s^{\star}\right)$, maximize their expected utility at extremal levels of diversification, i.e., $m^{\star}=M$;

- banks with large enough share of asset invested in the external assets $\left(s_{0} \geq s^{\star}\right)$, maximize their expected utility at intermediate levels of diversification, i.e., $1<m^{\star}<M$.

Formally, this is stated in the following proposition.

Proposition 5. Given the probability interval $\Omega_{p}:=\left[0, \frac{1}{2}\right)$ and under the existence of two possible states of the world where asset returns either follow a normally distributed downtrend (or uptrend) with mean equal to $\mu^{-}$(or $\mu^{+}$) and variance $\sigma$, the banks' expected utility maximization problem, as formalized in Eq. (24), leads to the following alternative solutions:

I. If $s_{0}<s^{*}$, the expected utility $\mathbb{E} U\left(\Pi_{m}\right)$ is maximized when $m^{\star}=M$. Then, $\mathbb{E} U\left(\Pi_{m}\right) \leq \mathbb{E} U\left(\Pi_{m^{\star}}\right)$, for all $m<M$.

II. if $s_{0} \geq s^{*}$, there exists a subinterval $\Omega_{p^{\star}} \subset \Omega_{p}$ s.t., to each $p^{\star} \in \Omega_{p^{\star}}$ corresponds an optimal level of diversification $m^{\star}$ in the open ball $B\left(\frac{1+M}{2}, r\right)=\left\{m^{\star} \in \mathbb{R} \mid d\left(m^{\star}, \frac{1+M}{2}\right)<r\right\}$ with center $\frac{1+M}{2}$ and radius $r \in[0, \alpha]$ where $\alpha=f(q, g)$. Then, $\mathbb{E} U\left(\Pi_{m}\right) \leq \mathbb{E} U\left(\Pi_{m^{\star}}\right)$, for all $m \notin B\left(\frac{1+M}{2}, r\right)$.

Proof: Appendix A.

Proposition 5 II. states that the optimal diversification can be an interior solution. ${ }^{13}$ Namely, when banks maximize their MV utility they may choose an intermediate level of diversification, viz., $1<m^{\star}<M . m^{\star}$ is the unique optimal solution and its level depends on the market size and on the likelihood of incurring in a negative or positive trend.

In the words of Haldane (2009), we show that diversification is a double-edged strategy. Values of $m \gtrless m^{\star}$ are second-best choices. Precisely, by increasing $m$ to approach $m^{\star}$ from below, banks increase their utility. However, by increasing $m$ beyond $m^{\star}$, banks decrease their utility. In summary, the MV utility exhibits inverse U-shaped non-monotonic behavior with respect to $m$. These results hold under the market structure described in the previous sections.

\footnotetext{
13 As a robustness check, we tested the validity of the proposition for different values of $p$. Results are robust also for low values of the probability of downtrend.
} 
a

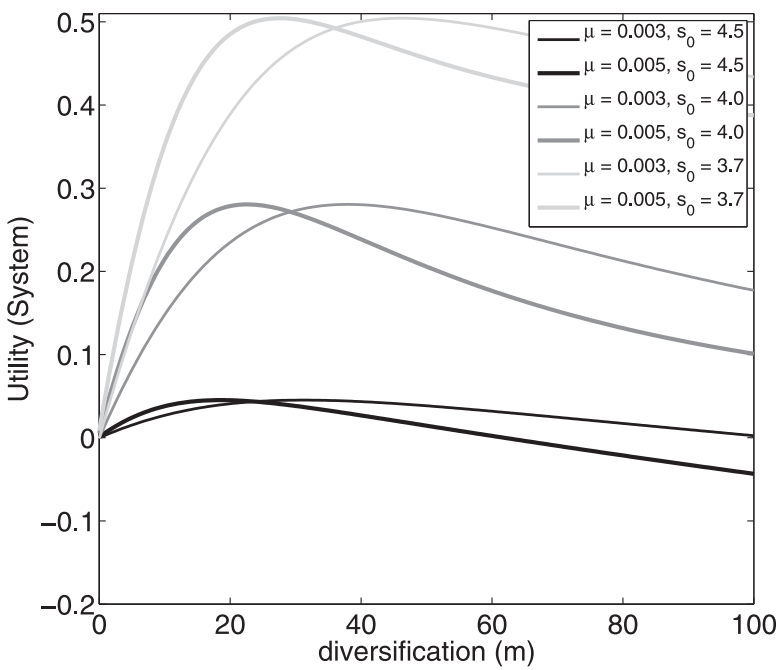

b

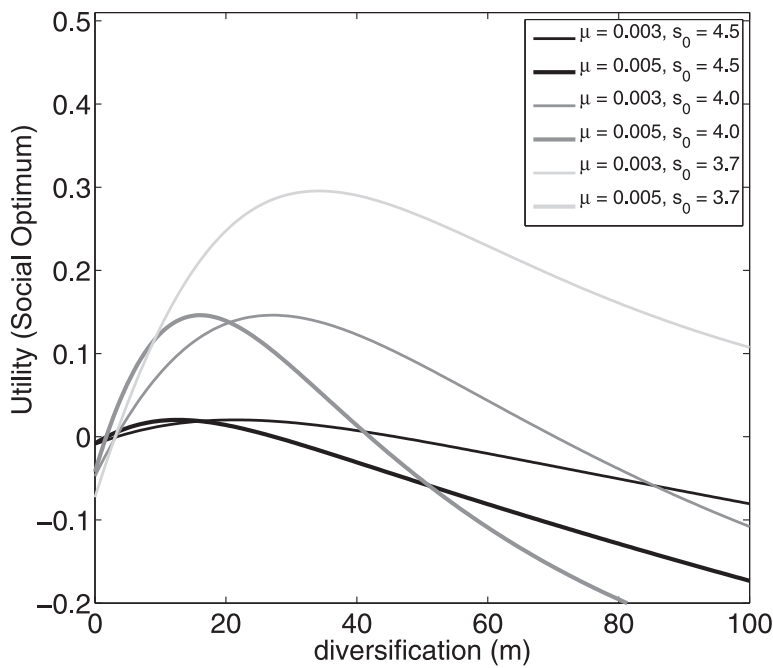

Fig. 7. Expected MV Utility for different levels of risk diversification. Fixed downtrend probability, $p=0.4$. The figure compares the expected MV utility of the banking system vs. the expected MV utility in the social optimum case. The curves represent different initial asset values, $s_{0}$, and different drifts, $\mu$. (a) Exp. MV Utility of the banking system $\mathbb{E} U\left(\Pi_{m}\right)$. (b) Exp. MV Utility in the social optimum case $\mathbb{E} U_{r}\left(\Pi_{m}\right)$. Parameters: $\sigma^{2}=0.5, r_{f}=0.001, \varepsilon=0.1$, $\lambda=0.1, \beta=0.2, l=0.5, k=2, m \in\{1, \ldots, 100\}, s_{0} \in\{3.7,4,4.5\}, w=1,\left|\mu^{+}\right|=\left|\mu^{-}\right| \in\{0.003,0.005\}$.

a

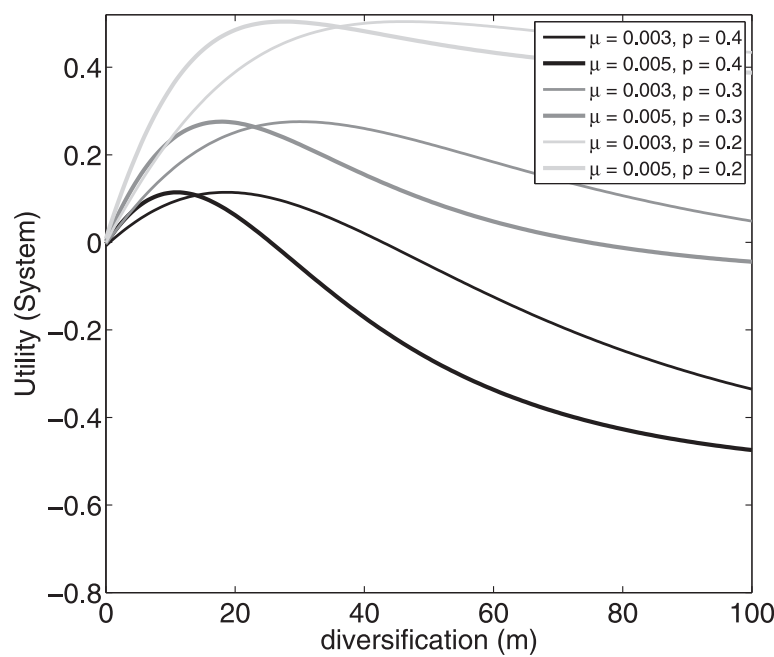

b

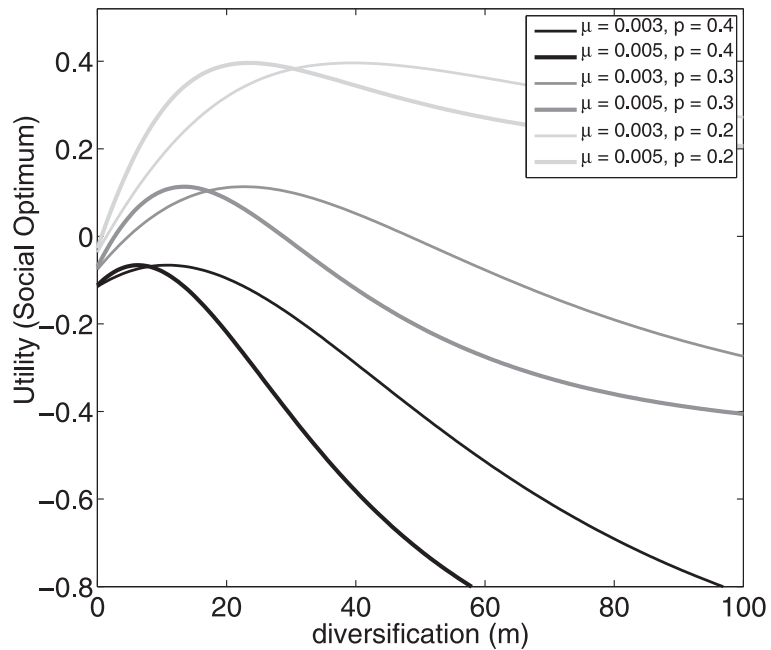

Fig. 8. Expected MV Utility for different levels of risk diversification. Fixed initial asset value, $s_{0}=3.69$. The figure compares the expected MV utility of the banking system vs. the expected MV utility in the social optimum. The curves represent different probabilities of downtrend, $p$ and different drifts, $\mu$. (a) Exp. MV Utility of the banking system $\mathbb{E} U\left(\Pi_{m}\right)$. (b) Exp. MV Utility in the social optimum $\mathbb{E} U_{r}\left(\Pi_{m}\right)$. Parameters: $\sigma^{2}=0.5, r_{f}=0.001, \varepsilon=0.1, \lambda=0.1$, $\beta=0.2, l=0.5, k=2, m \in\{1, \ldots, 100\}, w=1, p \in\{0.2,0.3,0.4\},\left|\mu^{+}\right|=\left|\mu^{-}\right| \in\{0.003,0.005\}$.

Briefly, banks are fully rational agents with incomplete information about the future state of the world. There are no transaction costs, or market asymmetries. Market returns exhibit a bimodal distribution. There are negative externalities on the interbank arising from the fact that banks' interbank assets are negatively affected when a counterparty's leverage increases. In our model, this externality is internalized by each bank since their utility function is computed by using a default probability model that accounts for the contagion from counterparties.

For a fixed probability $p$, Fig. 7a shows how the utility changes for different levels of diversification $m$, different magnitudes of the trend and different initial asset values, $s_{0}$. Instead, for a fixed initial asset value $s_{0}$, Fig. 8a shows how the utility changes for different levels of diversification $m$, different magnitudes of the trend and different probability of downtrend, $p$. Notice that, $m$ enters into the maximization problem via Eq. (15). In particular, the portfolio volatility decreases with $m$. Therefore, an outward movement along the $x$-axis in Figs. 7 and 8, i.e., increasing $m$, is equivalent to a market condition where the volatility of the assets is low, $\sigma=\sigma_{\text {low }}$. 
Conversely, an inward movement along the $x$-axis in Figs. 7 and 8, i.e., decreasing $m$, is equivalent to a market condition where the volatility of the assets is high, $\sigma=\sigma_{\text {high }}$. To conclude, one might observe that if banks are already in $m^{\star}$, an abrupt increases in the volatility of the assets (equivalent to an inward movement from $m^{\star}$ ) decreases the utility of the banks. ${ }^{14}$ In an optimization problem similar to our own, but with endogenous equilibrium asset pricing, Danielsson and Zigrand (2008) show that an increase in the volatility of both assets and portfolios can be generated by imposing strict risk-sensitive constraints of the VaR type.

\subsection{Conditional systemic default probability}

In this section, we analyze the probability of a systemic default conditional to the default of any one bank $j$, defined as $\mathbb{P}\left(\phi \geq 1 \mid \phi_{j} \geq 1\right)$. In particular, two basic properties hold. If banks do not have interbank exposures and are not diversified on their external assets, the conditional systemic default probability tends to zero for large number of banks, i.e. banks never default together.

On the contrary, when banks do have interbank exposure and are fully diversified in external assets, the conditional systemic default probability is identically one, i.e. banks always default together. Formally:

Proposition 6. Denote the probability of a systemic event conditional to the default of bank $j$ by $\mathbb{P}\left(\phi \geq 1 \mid \phi_{j} \geq 1\right)$. For any value of interbank exposure $1 \geq w \geq 0$ and some $j \in\{1, \ldots, N\}$, it holds:

If $m=1: \mathbb{P}\left(\phi \geq 1 \mid \phi_{j} \geq 1\right) \rightarrow 0$ for $n \rightarrow \infty$;

If $m=M: \mathbb{P}\left(\phi \geq 1 \mid \phi_{j} \geq 1\right)=1$ for $n \rightarrow \infty$.

Proof: Appendix A.

In Appendix C, we perform a set of Monte Carlo simulations in order to verify the above Proposition in the context of $n$ banks and to investigate how the conditional probability of systemic default varies for intermediate levels of diversification $m$ when banks hold overlapping portfolios. The conditional probability of simultaneous defaults will play an important role in the tension between individual banks and socially optimal diversification that is analyzed in the following section.

\section{Private incentives vs. social optimum}

In the following, we assume that there are social costs associated to the default of one or more banks due to negative externalities to the real economy that limited-liability banks commonly do not account for. We also assume that it is more costly to restore the functioning of the market in case of simultaneous defaults than in the case of isolated defaults. We intentionally leave the definition of social costs general as it may depend on the characteristics of the financial system under analysis. Consider a sophisticated bank that, differently from the other banks, internalizes the social costs. For simplicity we define such case as "social optimum". We want to formulate the utility maximization problem in the "social optimum" and compare it to the utility maximization problem of individual banks in Eq. $(24)^{15}$.

Let $K$ be the number of simultaneously defaulting banks. We make the following assumptions:

Assumption 1. The total loss to be accounted in the case of social optimum in downtrend periods is a monotonically increasing function $f\left(k, \pi^{-}\right):=k \pi^{-}$of: (i) the expected number $k$ of bank crashes given a collapse of at least one bank $\mathbb{E}(K \mid K \geq 1)=k$, (ii) the magnitude of the loss $\pi^{-}$.

\subsection{Social optimum utility function}

Therefore, the social optimum's utility maximization problem is as follows,

$$
\begin{array}{rl}
\max _{m} & \mathbb{E} U_{r}\left(\Pi_{m}\right)=\mathbb{E}_{r}\left(\Pi_{m}\right)-\frac{\lambda \sigma_{r}^{2}\left(\Pi_{m}\right)}{2} \\
\text { s.t.: } & \left\{\begin{array}{l}
1 \leq m \leq M \\
l>0 \\
s^{-}<s_{0}<s^{+} \\
p \mu_{s}^{-}+(1-p) \mu_{s}^{+}>0 \\
k>1
\end{array}\right.
\end{array}
$$

\footnotetext{
${ }^{14}$ This result could surprise as a possible explanation for the empirical findings that solvency condition across financial institutions, in the recent US 2007-08 crises, has been driven by an increase in the volatility of the firm's assets, Atkeson et al. (2013).

${ }^{15}$ In the context of our model, we analyze the misalignments between private incentives and social optimal level of diversification, but we do not formalize the problem of a social planner who intends to maximize social welfare by choosing the extent of diversification in banks' exposures to external assets. Rather, we assess social optimality by comparing the optimal level of diversification chosen by single banks with that of a bank internalizing the negative externalities caused by banks' failures in the system.
} 
a

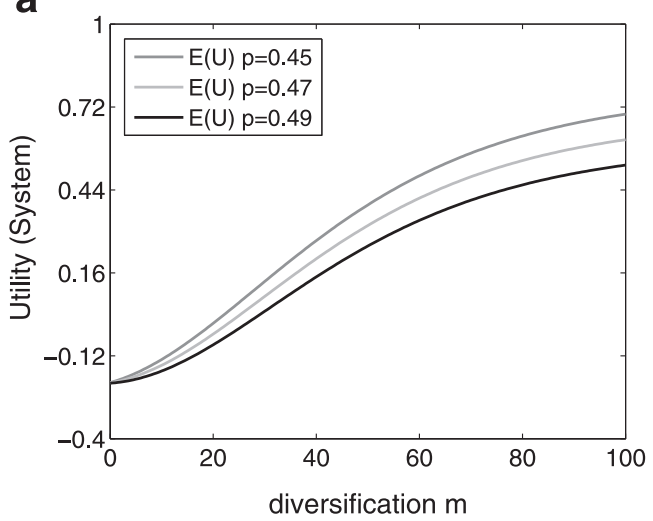

b

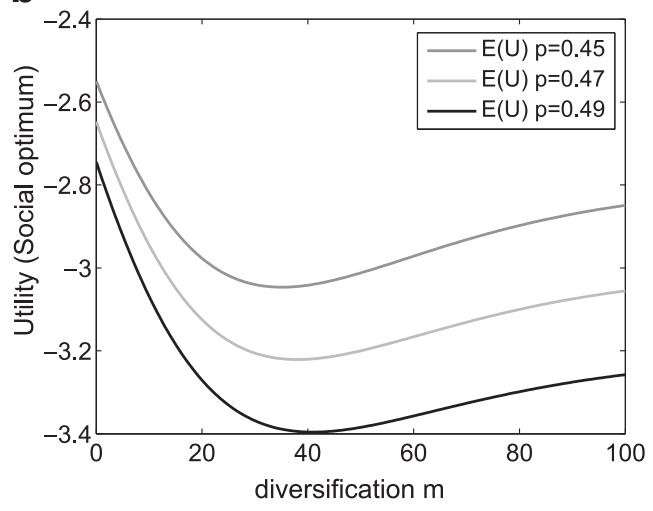

Fig. 9. Tension between individual bank and social optimum Comparison between expected utility function of (a) individual banks (b) and social optimum. Parameters: $p \in\{0.45,0.47,0.49\}, \sigma^{2}=0.5, r_{f}=0.001, \epsilon=0.1, \lambda=0.1, \beta=0.2, w=1, l=0.5, k=5, m \in\{1, \ldots, 100\} s_{0}=1.5,\left|\mu^{+}\right|=\left|\mu^{-}\right|=0.003$.

$$
\text { with }\left\{\begin{array}{c}
\mathbb{E}_{r}\left(\Pi_{m}\right)=p\left[q k \pi^{-}+(1-q) \pi^{+}\right]+(1-p)\left[g \pi^{-}+(1-g) \pi^{+}\right] \\
\sigma_{r}^{2}\left(\Pi_{m}\right)=p\left[q\left(k \pi^{-}-\mathbb{E}\left(\Pi_{m}\right)\right)^{2}+(1-q)\left(\pi^{+}-\mathbb{E}\left(\Pi_{m}\right)\right)^{2}\right] \\
+(1-p)\left[g\left(\pi^{-}-\mathbb{E}\left(\Pi_{m}\right)\right)^{2}+(1-g)\left(\pi^{+}-\mathbb{E}\left(\Pi_{m}\right)\right)^{2}\right] .
\end{array}\right.
$$

Notice that, with respect to the optimization in Eq. (24), the social optimum is subject to the additional constraint $k>1$ that amplifies both the expected loss and its variance. In summary, we compare the general solution of the maximization problem in Eq. (24) with that in Eq. (25).

\subsection{The tension between individual and social optimum's diversification}

The results shown in the previous sections imply the emergence of a tension between the incentives of the individual banks and the social optimum, a finding that is important also in terms of policy implications. Consider the following (not implausible) scenario in which the downtrend is less probable than the uptrend and yet its probability is not negligible. Then, banks will prefer a maximal diversification. So doing, will hold completely correlated portfolios and they will tend to default altogether. This implies a very high social cost in the social optimum, since banks would have instead very low diversification in order to keep defaults less correlated. The tension between private and public incentive is extreme in this case. In the following Proposition we show that the optimal level of diversification for the social optimum, $m^{r}$, is lower than the level that is desirable for individual banks, $m^{\star}$.

Proposition 7. If banks do not internalize the impact of social costs in the case of simultaneous defaults, in equilibrium the banking system is over-diversified on external assets w.r.t. to the level of diversification that is socially desirable:

$$
m^{\star} \geq m^{r}
$$

and

$$
m^{r}<\infty
$$

$\forall s_{0} \in\left(s^{-}, s^{+}\right)$.

Proof: Appendix A.

To illustrate the result in Proposition 7 we compare Fig. 7a with Fig. 7b and Fig. 8a with Fig. 8b. We can observe that the tension between individual and social incentives leads to a banking system that maximizes its expected utility at a level of diversification higher than the one maximizing the expected utility in the social optimum, viz. $m^{\star} \geq m^{r}$. Moreover, there is a range of parameters where the tension is maximal because $m^{\star}=M$ and $m^{r}=1$. This is illustrated in Fig. 9 and in the following numerical result. See Appendix C.

Remark 3. If the external asset price is small enough, $s_{0}<s^{\star}$ (see Proposition 5.I), there exists a range of $p$, such that $\{p \in \mathbb{R} \mid d(p, 0.5)<r\}$ with $r>0$, in which the expected utility of the banking system is maximized for the maximum level of diversification while the expected utility in the social optimum is maximized for the minimum level of diversification.

$$
m^{\star}=M>m^{r}=1 .
$$

See Appendix C. 


\section{Concluding remarks}

This paper develops a new modeling framework in order to investigate the probability of default in a system of banks holding claims on each others as well as overlapping portfolios due to bank diversification in a market with a finite number of external assets.

While our framework, uniquely combining together network and portfolio theories, allows for the investigation of several cross-disciplinary questions, we focus on the core of tightly knit banks, i.e., $w=1$. In this setting, we study how the probability of a systemic default depends on the level of diversification in external assets.

To incorporate the network effect, we measure the performance of portfolio diversification by its impact to banks default probabilities and expected utility, rather than by its impact on the asset portfolio volatility. By doing that, we extend the literature focusing on the hedging efficiency in the context of portfolio management alone, e.g., Markowitz (1952), Johnson and Shannon (1974). Indeed, the network of interbank claims exposes each individual bank to shocks on those external assets held by its counterparties. The network amplifies the effect of the negative trend, when it occurs, and the impact of shocks when banks have largely overlapping portfolios due to extensive diversification on external assets. In general terms, our paper (implicitly) argues that firms optimal hedging choice should incorporate firms balance sheet structure as a whole, such as counterparty exposures and leverage ratio.

As a second contribution, this paper offers a complementary approach with respect to the strand of works (on the valuation of distressed assets) building on Eisenberg and Noe (2001). In those works the default process is considered only at the maturity of the interbank contracts. Differently, in this paper the values of the interbank claims are dependent among each others and evolve over time as a system of stochastic processes. By so doing, the contagion propagated through the network is internalized by the banks even before the maturity of the contracts.

A third contribution consists of investigating the optimal diversification strategy of banks in the face of opposite and persistent economic trends that are unknown to banks. Maximal risk diversification minimizes the default probability if the economic trend happens to be positive, because it reduces the downside risk. In contrast, a minimal risk diversification minimizes the default probability when the economic trend happens to be negative because gives the chance to "gamble for resurrection". If banks, for various constraints, are not able to change diversification strategy during a certain time, then we could intuitively think that the optimal level of risk diversification is always interior because it must be a convex combination of the two optima. The exact level of interior optimal diversification should depend on the probability of the market trend to be positive or negative. However, this intuition suffers from a logical fallacy. In fact, maximizing a convex combination of functions is not equivalent to take the convex combination of the maxima. Accordingly, we show that optimal risk diversification is interior under certain conditions, but, in general, it is not interior. We find that banks' optimal diversification is extremal when the exposure to the external assets is not too large and it is interior otherwise.

Notice that banks do internalize the negative externalities resulting from banks holding claims on counterparties' liabilities, the value of which is affected by counterparties leverage. Indeed, the default probability, used by banks when computing the optimal diversification, takes into account contagion.

As a fourth contribution, this paper offers an analysis of the tension arising between banks' incentives and social optimum. For the largest range of the parameters, bank's optimal choice leads to full (or very high) diversification across the external assets. However, this also leads to banks having highly correlated portfolios and suffering simultaneous defaults. The later may engender high social costs for the real economy. When those social costs are internalized, the socially desirable level of diversification is lower than the level chosen by the banking system.

Our work could be extended in a number of ways. In particular, one could study both the effect of diversification across counterparties and external assets. Notice that, in order to better isolate the effectiveness of diversification in mitigating idiosyncratic risks to which banks are exposed via external assets holding, in our setting the interbank diversification is fixed and homogeneous. However, one could introduce some heterogeneity in the balance sheet structure and in the portfolio holdings of the banks and answer the question whether external diversification and interbank diversification are substitutes or complements.

Overall, an important point stemming from our analysis lies in the recognition that social optimum is not to target a specific diversification level of risk but rather to manage the trade-off between the social losses from defaults (because of excessive risk spreading in economic downturn) and the social costs of avoiding defaults (because of excessive risk diversification in economic booms).

\section{Acknowledgments}

We are grateful to Andrea Collevecchio, Co-Pierre Georg, Martino Grasselli, Christian Julliard, Helmut Helsinger, Rahul Kaushik, Iman van Lelyveld, Moritz Müller, Paolo Pellizzari, Loriana Pelizzon, Didier Sornette, Claudio J. Tessone, Frank Schweitzer, Joseph Stiglitz, Jean-Pierre Zigrand and participants at various seminars and conferences where preliminary versions of this paper have been presented.

The first version of this work was written while S.B. and P.T. were employed at the Chair of Systems Design at ETH Zurich, lead by Frank Schweitzer, to whom they are thankful for support and guidance. The authors acknowledge financial support from the ETH Competence Center "Coping with Crises in Complex Socio-Economic Systems" [CHIRP 1 grant no. CH1-0108-2], the European FET Open Project "FOC" [grant no. 255987], and the SNSF project "OTC Derivatives and Systemic Risk 
in Financial Networks" [grant no. CR12I1-127000/1]. The support of the Economic and Social Research Council (ESRC) is gratefully acknowledged [grant number ES/K002309/1]. A previous version of this paper appears in the CCSS Working Paper No. 11-001, ETH Zurich. S.B. acknowledge the Swiss National Fund Professorship grant no. PP00P1-144689. S.B. and A.D. acknowledges support from: FET Project SIMPOL nr. 610704.

\section{Appendix A. Proofs}

Remark 1. If $f$ or $\phi_{i} \rightarrow 1$ the marked-to-market value if interbank liabilities is such that $\hat{\eta}_{i}>0$, the implicit recovery rate is:

$$
\delta=\left\{\begin{array}{l}
1 \text { in case of no default } \\
\frac{1+r_{f}}{1+r f+\beta} \quad \text { in case of default }
\end{array}\right.
$$

Proof. The recovery rate is the proportion of face value that is recovered through bankruptcy procedures in the event of a default. Therefore, the general formula for the discounted recovery rate $\hat{\delta}$ is

$$
\begin{aligned}
\hat{\delta} & =\frac{l_{i}}{\left(1+r_{f}+\beta \phi_{i}\right)}\left(\frac{1}{l_{i}}\right) \\
& =\frac{1}{\left(1+r_{f}+\beta \phi_{i}\right)} .
\end{aligned}
$$

That is

$$
\hat{\delta}= \begin{cases}\frac{1}{1+r f} & \text { in case of no default } \\ \frac{1}{1+r f+\beta} & \text { in case of default }\end{cases}
$$

The actual recovery rate at the maturity of the debt is $\delta=\hat{\delta}\left(1+r_{f}\right)$. That is

$$
\delta=\left\{\begin{array}{l}
1 \text { in case of no default } \\
\frac{1+r_{f}}{1+r f+\beta} \quad \text { in case of default }
\end{array}\right.
$$

Remark 2. Bank's leverage defined as in Eq. (10) is a second order polynomial equation in in $\mathbf{\Phi}$ :

$$
\boldsymbol{\Phi} \mathbf{I} \beta \boldsymbol{\Phi}+\boldsymbol{\Phi} \mathbf{I R}-\mathbf{L}(\mathbf{W H})^{-1} \beta \boldsymbol{\Phi}+\boldsymbol{\Phi}=\mathbf{L}(\mathbf{W H})^{-1} \mathbf{R}
$$

where $\quad \mathbf{H}:=\operatorname{diag}\left(\eta_{1}, \eta_{2}, \ldots, \eta_{M}\right) ; \quad \mathbf{I}:=\mathbf{Z V}(\mathbf{W H})^{-1} \quad$ and $\quad \boldsymbol{\Phi}:=\operatorname{diag}\left(\phi_{1}, \phi_{2}, \ldots, \phi_{N}\right) ; \quad \mathbf{L}:=\operatorname{diag}\left(l_{1}, l_{2}, \ldots, l_{N}\right) ;$ $\mathbf{V}:=\operatorname{diag}\left(v_{1}, v_{2}, \ldots, v_{M}\right) ; \mathbf{R}:=\operatorname{diag}(R, R, \ldots, R)$ with $R=1+r_{f} ; \mathbf{W}:=\left[w_{i k}\right]_{N \times N} ; \mathbf{Z}:=\left[z_{i j}\right]_{N \times M}$.

Proof. The leverage at banking system level can be derived from Eq. (10) and rewritten as:

$$
l_{i}=\phi_{i} \times\left(\sum_{j} z_{i j} v_{j}+\sum_{k} w_{i k} \eta_{k} /\left(1+r_{f}+\beta \phi_{k}\right)\right) .
$$

In vector notation, Eq. (A.3) is equivalent to

$$
\mathbf{L}=\boldsymbol{\Phi} \times\left[\mathbf{Z V}+(\mathbf{R}+\beta \boldsymbol{\Phi})^{-1} \mathbf{W H}\right]
$$

that we explicit for $\boldsymbol{\Phi}$ :

$$
\begin{aligned}
& \mathbf{L}=\boldsymbol{\Phi}\left[\mathbf{Z V}+(\mathbf{R}+\beta \boldsymbol{\Phi})^{-1} \mathbf{W H}\right] \\
& \mathbf{L}=\boldsymbol{\Phi} \mathbf{Z V}+\boldsymbol{\Phi}(\mathbf{R}+\beta \boldsymbol{\Phi})^{-1} \mathbf{W H} \\
& \mathbf{L}(\mathbf{W H})^{-1}=\boldsymbol{\Phi Z V}(\mathbf{W H})^{-1}+\boldsymbol{\Phi}(\mathbf{R}+\beta \boldsymbol{\Phi})^{-1} \mathbf{W H}(\mathbf{W H})^{-1} \\
& \mathbf{L}(\mathbf{W H})^{-1}(\mathbf{R}+\beta \boldsymbol{\Phi})=\boldsymbol{\Phi Z V}(\mathbf{W H})^{-1}(\mathbf{R}+\beta \boldsymbol{\Phi})+\boldsymbol{\Phi}(\mathbf{R}+\beta \boldsymbol{\Phi})^{-1}(\mathbf{R}+\beta \boldsymbol{\Phi}) \\
& \mathbf{L}(\mathbf{W H})^{-1} \mathbf{R}+\mathbf{L}(\mathbf{W H})^{-1} \beta \boldsymbol{\Phi}=\boldsymbol{\Phi Z V}(\mathbf{W H})^{-1}(\mathbf{R}+\beta \boldsymbol{\Phi})+\boldsymbol{\Phi} \\
& \boldsymbol{\Phi} \mathbf{I} \beta \boldsymbol{\Phi}+\boldsymbol{\Phi} \mathbf{I R}-\mathbf{L}(\mathbf{W H})^{-1} \beta \boldsymbol{\Phi}+\boldsymbol{\Phi}=\mathbf{L}(\mathbf{W H})^{-1} \mathbf{R}
\end{aligned}
$$

where $\mathbf{I}:=\mathbf{Z V}(\mathbf{W H})^{-1}$.

Proposition 1. Consider the default conditions in Eq. (22) and assume that external assets dynamics follows a timehomogeneous process with the same drift and same variance. If external assets are uncorrelated, $\mathbb{P}($ default $)$, can be expressed as

$$
\mathbb{P}(\text { default })=\left(\int_{s_{0}}^{s^{+}} d s \psi(x)\right) /\left(\int_{s^{-}}^{s^{+}} \mathrm{d} x \psi(x)\right), \text { where } \psi(x)=\exp \left(\int_{0}^{x}-\frac{2 \hat{\mu}}{\hat{\sigma}^{2}} \mathrm{~d} s\right)
$$


and has the following closed form solution:

$$
\mathbb{P}(\text { default })=\left(\exp \left[-\frac{2 \hat{\mu} s_{0}}{\hat{\sigma}^{2}}\right]-\exp \left[-\frac{2 \hat{\mu} s^{+}}{\hat{\sigma}^{2}}\right]\right) /\left(\exp \left[-\frac{2 \hat{\mu} s^{-}}{\hat{\sigma}^{2}}\right]-\exp \left[-\frac{2 \hat{\mu} s^{+}}{\hat{\sigma}^{2}}\right]\right) .
$$

Proof. In compact form, Eq. (19) reads as $\phi=f\left(s, l, r_{f}, \beta\right)$. The dynamics of $\phi$ depends directly on the dynamics of $s$ in Eq. (15) because both $r_{f}$ and $l$ are real constant and $\beta$ is a coefficient. Therefore, to derive the systemic default probability $\mathbb{P}(\phi \geq 1)$ in a close form, one could find, via Ito's Lemma, the dynamics of $\phi$ from the dynamics of $s$ and observe whether $\phi(0) \in(\varepsilon, 1)$ exits after time $t \geq 0$ through the upper default boundary fixed at one. However, $f$ is highly non linear in $s$, see Eq. (19). Thus, it is convenient to derive the systemic default probability directly from the dynamics of $s$ by mapping the sample space of $\phi$ into the sample space of $s$. Since the partial derivative of $f$ with respect to $s$, i.e, $\frac{\partial f}{\partial s}=$ $-l /[s+(l w /(\beta \phi+R))]^{2}$, is negative for all $s$ and for any value of $r_{f}, l$ in their range of variation, the Inverse Function Theorem implies that $f$ is invertible on $\mathbb{R}^{+}$:

$$
f^{-1}(\phi)=\frac{l(\beta \phi+R-\phi w)}{\phi(\beta \phi+R)}, \quad \text { s.t. } \quad\left(f^{-1}\right)^{\prime}(\phi)=\frac{1}{f^{\prime}(s)} .
$$

Observe that, by definition, the value of external assets cannot be negative, $s \in \mathbb{R}^{+}$. Given the above result, we obtain the following mapping between the values of $\phi$ and $s$ :

$$
\begin{cases}f(s)=1 & \text { iff } f^{-1}(\phi)=\frac{l(\beta+R-w)}{\beta+R}:=s^{-} \\ f(s)=\varepsilon & \text { iff } f^{-1}(\phi)=\frac{l(\beta \varepsilon+R-w \varepsilon)}{\beta \varepsilon^{2}+R \varepsilon}:=s^{+}\end{cases}
$$

Hence, the systemic default probability can be defined also with respect to $s$ :

$$
\mathbb{P}(\text { default })=\mathbb{P}(\phi \geq 1) \equiv \mathbb{P}\left(s \leq s^{-}\right) .
$$

This is the probability that $s$, initially at an arbitrary level $s(0):=s_{0} \in\left(s^{-}, s^{+}\right)$, exits through the lower default boundary $s^{-}$ after time $t \geq 0$. From Gardiner (1985), $\mathbb{P}\left(s \leq s^{-}\right)$has the following explicit form:

$$
\mathbb{P}\left(s \leq s^{-}\right)=\left(\int_{s_{0}}^{s^{+}} d s \psi(x)\right) /\left(\int_{s^{-}}^{s^{+}} \mathrm{d} x \psi(x)\right) .
$$

with $\psi(x)=\exp \left(\int_{0}^{x}-\frac{2 \hat{\mu}}{\hat{\sigma}^{2}} \mathrm{~d} s\right)$. Eq. (A.5) has the following closed form solution:

$$
\mathbb{P}\left(s \leq s^{-}\right)=\left(\exp \left[-\frac{2 \hat{\mu} s_{0}}{\hat{\sigma}^{2}}\right]-\exp \left[-\frac{2 \hat{\mu} s^{+}}{\hat{\sigma}^{2}}\right]\right) /\left(\exp \left[-\frac{2 \hat{\mu} s^{-}}{\hat{\sigma}^{2}}\right]-\exp \left[-\frac{2 \hat{\mu} s^{+}}{\hat{\sigma}^{2}}\right]\right),
$$

with $\hat{\mu}=p \mu^{+}+(1-p) \mu^{-}$and $\hat{\sigma}^{2}=p\left[\left(\mu^{+}-\hat{\mu}\right)^{2}+\frac{\sigma^{2}}{m}\right]+(1-p)\left[\left(\mu^{-}-\hat{\mu}\right)^{2}+\frac{\sigma^{2}}{m}\right]$.

Proposition 2. The conditional systemic default probability, conditional to a given market trend has the following closed form solutions:

$$
\left\{\begin{array}{l}
q=\left(\exp \left[-\frac{\left(2 \mu^{-}\right) s_{0}}{\sigma^{2} / m}\right]-\exp \left[-\frac{\left(2 \mu^{-}\right) s^{+}}{\sigma^{2} / m}\right]\right) /\left(\exp \left[-\frac{\left(2 \mu^{-}\right) s^{-}}{\sigma^{2} / m}\right]-\exp \left[-\frac{\left(2 \mu^{-}\right) s^{+}}{\sigma^{2} / m}\right]\right), \\
g=\left(\exp \left[-\frac{\left(2 \mu^{+}\right) s_{0}}{\sigma^{2} / m}\right]-\exp \left[-\frac{\left(2 \mu^{+}\right) s^{+}}{\sigma^{2} / m}\right]\right) /\left(\exp \left[-\frac{\left(2 \mu^{+}\right) s^{-}}{\sigma^{2} / m}\right]-\exp \left[-\frac{\left(2 \mu^{+}\right) s^{+}}{\sigma^{2} / m}\right]\right) .
\end{array}\right.
$$

Proof. From the proof of Proposition 1, we can derive immediately that during a downtrend, Eq. (A.5) yields the conditional default probability given a downtrend

$$
q:=\mathbb{P}\left(\text { de fault } \mid \mu^{-}\right)=\mathbb{P}\left(s \leq s^{-} \mid \mu^{-}\right)
$$

with the following closed form solution:

$$
q=\left(\exp \left[-\frac{\left(2 \mu^{-}\right) s_{0}}{\sigma^{2} / m}\right]-\exp \left[-\frac{\left(2 \mu^{-}\right) s^{+}}{\sigma^{2} / m}\right]\right) /\left(\exp \left[-\frac{\left(2 \mu^{-}\right) s^{-}}{\sigma^{2} / m}\right]-\exp \left[-\frac{\left(2 \mu^{-}\right) s^{+}}{\sigma^{2} / m}\right]\right) .
$$

During an uptrend, Eq. (A.5) yields the conditional default probability given an uptrend:

$$
g:=\mathbb{P}\left(\text { de fault } \mid \mu^{+}\right)=\mathbb{P}\left(s \leq s^{-} \mid \mu^{+}\right)
$$

with the following closed form solution:

$$
g=\left(\exp \left[-\frac{\left(2 \mu^{+}\right) s_{0}}{\sigma^{2} / m}\right]-\exp \left[-\frac{\left(2 \mu^{+}\right) s^{+}}{\sigma^{2} / m}\right]\right) /\left(\exp \left[-\frac{\left(2 \mu^{+}\right) s^{-}}{\sigma^{2} / m}\right]-\exp \left[-\frac{\left(2 \mu^{+}\right) s^{+}}{\sigma^{2} / m}\right]\right) .
$$


Proposition 3. Consider a debt-financed portfolio subject to a given default threshold as in the model described so far. Then, the default probability decreases with risk diversification in case of asset prices uptrend and increases with diversification in case of asset prices downtrend.

Proof. We provide an asymptotic analysis that explains the results presented in Proposition 3. Let rewrite Eqs. (A.7) and (A.8) as

$$
\begin{aligned}
q & =\frac{M_{(-)}^{s_{0}}-M_{(-)}^{s^{+}}}{M_{(-)}^{s^{-}}-M_{(-)}^{s^{+}}} \\
& =M_{(-)}^{\left(s_{0}-s^{+}\right)}\left[M_{(-)}^{\left(s^{-}-s^{+}\right)}-1\right]-\frac{1}{M_{(-)}^{\left(s^{-}-s^{+}\right)}-1} \\
g & =\frac{M_{(+)}^{s_{0}}-M_{(+)}^{s^{+}}}{M_{(+)}^{S^{-}}-M_{(+)}^{S^{+}}} \\
& =M_{(+)}^{\left(s_{0}-s^{+}\right)}\left[M_{(+)}^{\left(s^{-}-s^{+}\right)}-1\right]-\frac{1}{M_{(+)}^{\left(s^{-}-s^{+}\right)}-1}
\end{aligned}
$$

with $M_{(-)}=\exp \left[\frac{-\left(2 \mu^{-}\right) m}{\sigma^{2}}\right]$ and $M_{(+)}=\exp \left[\frac{-\left(2 \mu^{+}\right) m}{\sigma^{2}}\right]$. Then, the following result is straightforward:

$$
\left\{\begin{array}{l}
\lim _{m \rightarrow+\infty} q=0-(-1)=1 \\
\lim _{m \rightarrow+\infty} g=0-0=0
\end{array}\right.
$$

To conclude, for any arbitrary small number $\epsilon \in(0,1)$

$$
\exists \bar{m}>1 \mid(q-g)>1-\epsilon \quad \forall m>\bar{m}
$$

Proposition 4. Assume homogeneity in the banks' capital structure and the presence of a network of interbank exposures among banks. For a given default threshold, ceteris paribus, the leverage of an individual bank, measured in terms of its leverage $\phi$, increases non-linearly with the level of its borrowers leverage and proportionally to the level of its interbank exposures.

Proof. To prove Proposition 4, we study the impact of the leverage of direct counterparties of bank $i, \phi_{k}$ on Eq. (10):

$$
\phi_{i}=l_{i} /\left(\sum_{j} z_{i j} v_{j}+\sum_{k} w_{i k}\left(\frac{\eta_{k}}{1+r_{f}+\beta \phi_{k}}\right)\right) .
$$

Considering only the interbank assets:

$$
\hat{\eta}_{i}=\sum_{k} w_{i k}\left(\frac{\eta_{k}}{1+r_{f}+\beta \phi_{k}}\right) .
$$

We can prove the effect of the network on the leverage of banks simply by studying the sign and the relation between the derivative of $\hat{\eta}_{i}$ and $\phi_{k}$ :

$$
\frac{\partial \hat{\eta}_{i}}{\partial \phi_{k}}=-\frac{w_{i k} \eta_{k}}{\left(1+r_{f}+\beta \phi_{k}\right)^{2}}
$$

As it comes out, the higher is $\phi_{k}$ the lower will be the value of interbank assets, $\hat{\eta}_{i}$. The derivative is proportional to $w_{i k}$, hence the greater the value of the exposures with the counterparties, the larger will be the effect of others' leverage on bank $i$ interbank assets. We conclude simply noting that, as it is possible to see from Eq. (10), a decrease in the level of $\hat{\eta}_{i}$, induced by higher level of $\phi_{k}$ translates directly in higher level of $\phi_{i}$ for bank $i$, and hence of its leverage.

Proposition 5. Given the probability interval $\Omega_{p}:=\left[0, \frac{1}{2}\right)$ and under the existence of two possible states of the world where asset returns either follow a normally distributed downtrend (or uptrend) with mean equal to $\mu^{-}$(or $\mu^{+}$) and variance $\sigma$, the banks' expected utility maximization problem, as formalized in Eq. (24), leads to the following alternative solutions:

I. If $s_{0}<s^{*}$, the expected utility $\mathbb{E} U\left(\Pi_{m}\right)$ is maximized when $m^{\star}=M$. Then, $\mathbb{E} U\left(\Pi_{m}\right) \leq \mathbb{E} U\left(\Pi_{m^{\star}}\right)$, for all $m<M$.

II. if $s_{0} \geq s^{*}$, there exists a subinterval $\Omega_{p^{\star}} \subset \Omega_{p}$ s.t., to each $p^{\star} \in \Omega_{p^{\star}}$ corresponds an optimal level of diversification $m^{\star}$ in the open ball $B\left(\frac{1+M}{2}, r\right)=\left\{m^{\star} \in \mathbb{R} \mid d\left(m^{\star}, \frac{1+M}{2}\right)<r\right\}$ with center $\frac{1+M}{2}$ and radius $r \in[0, \alpha]$ where $\alpha=f(q, g)$. Then, $\mathbb{E} U\left(\Pi_{m}\right) \leq \mathbb{E} U\left(\Pi_{m^{\star}}\right)$, for all $m \notin B\left(\frac{1+M}{2}, r\right)$. 
Proof. Proposition 5.I

To prove Proposition 5.I, note first that the function to maximize, as formalized in Eq. (24), is not linear. Since what we are eventually interested in is the $\max$ of $\mathrm{U}$, it is not possible to switch in general the linear operator with the expectation operator. To show the point, let us decompose the expected utility function for the two scenarios as follows:

$$
\begin{aligned}
& U\left(\Pi_{m}\right)_{\mu^{-}}=q \pi^{-}+(1-q) \pi^{+}-\frac{\lambda}{2}\left\{q\left[\pi^{-}-\mathbb{E}(\pi)\right]^{2}+(1-q)\left[\pi^{+}-\mathbb{E}(\pi)\right]^{2}\right\} ; \\
& U\left(\Pi_{m}\right)_{\mu^{+}}=g \pi^{+}+(1-g) \pi^{+}-\frac{\lambda}{2}\left\{g\left[\pi^{-}-\mathbb{E}(\pi)\right]^{2}+(1-g)\left[\pi^{+}-\mathbb{E}(\pi)\right]^{2}\right\} ;
\end{aligned}
$$

Assuming for simplicity that $\lambda=0$, the derivative Eqs. (A.13a) and (A.13b) with respect to $m$, can be derived as follows:

$$
\begin{aligned}
& \frac{\partial U\left(\Pi_{m}\right)_{\mu^{-}}}{\partial m}=\frac{\partial q}{\partial m} \pi^{-}-\frac{\partial q}{\partial m} \pi^{+}=-\frac{\partial q}{\partial m}\left(\pi^{+}-\pi^{-}\right) ; \\
& \frac{\partial U\left(\Pi_{m}\right)_{\mu^{+}}}{\partial m}=\frac{\partial g}{\partial m} \pi^{-}-\frac{\partial g}{\partial m} \pi^{+}=-\frac{\partial g}{\partial m}\left(\pi^{+}-\pi^{-}\right) ;
\end{aligned}
$$

Furthermore, given that utility function $U\left(\Pi_{m}\right)_{\mu}$ is the combination of the functions $U\left(\Pi_{m}\right)_{\mu^{-}}$and $U\left(\Pi_{m}\right)_{\mu^{+}}$, it follows that its derivative is equal to the combination of the derivatives of Eqs. (A.14) and (A.15). Hence:

$$
\begin{aligned}
\frac{\partial U\left(\Pi_{m}\right)_{\mu}}{\partial m} & =p \frac{\partial U\left(\Pi_{m}\right)_{\mu^{-}}}{\partial m}+(1-p) \frac{\partial U\left(\Pi_{m}\right)_{\mu^{+}}}{\partial m} \\
& =p\left[-\frac{\partial q}{\partial m}\left(\pi^{+}-\pi^{-}\right)\right]+(1-p)\left[-\frac{\partial g}{\partial m}\left(\pi^{+}-\pi^{-}\right)\right] \\
& =p\left(-\frac{\partial q}{\partial m} \Delta \pi\right)-(1-p)\left(\frac{\partial g}{\partial m} \Delta \pi\right)
\end{aligned}
$$

where $\Delta \pi=\pi^{+}-\pi^{-}$. In the above equations, the derivatives of the default probabilities $q$ and $g$ are respectively positive and negative. To prove it, we rewrite Eqs. (A.7) and (A.8) as follows:

$$
\begin{array}{r}
q=\frac{\exp \left[-\left(2 m \mu^{-}\right)\left(s^{+}-s_{0}\right)\right]-1}{\exp \left[-\left(2 m \mu^{-}\right)\left(s^{+}-s^{-}\right)\right]-1} \\
g=\frac{\exp \left[-\left(2 m \mu^{+}\right)\left(s^{+}-s_{0}\right)\right]-1}{\exp \left[-\left(2 m \mu^{+}\right)\left(s^{+}-s^{-}\right)\right]-1}
\end{array}
$$

where, we discard the trivial case $\mu=0$, and w.l.g. we set $\sigma^{2}=1$. The partial derivatives of Eq. (A.16a) and (A.16b) with respect to $m$ are positive and negative, respectively:

$$
\begin{aligned}
& \frac{\partial q}{\partial m}=\frac{2 e^{-2 m \mu^{-}\left(s_{0}+s^{-}-s^{+}\right)} \mu^{-}\left(e^{2 m \mu^{-} s^{+}}\left(s^{-}-s_{0}\right)+e^{2 m \mu^{-} s^{-}}\left(s_{0}-s^{+}\right)+e^{2 m \mu^{-} s 0}\left(-s^{-}+s^{+}\right)\right)}{e^{2 m \mu^{-}\left(s^{+}-s^{-}\right)}}>0 ; \\
& \frac{\partial g}{\partial m}=\frac{2 e^{-2 m \mu^{+}\left(s_{0}+s^{-}-s^{+}\right)} \mu^{+}\left(e^{2 m \mu^{+} s^{+}}\left(s^{-}-s_{0}\right)+e^{2 m \mu^{+} s^{-}}\left(s_{0}-s^{+}\right)+e^{2 m \mu^{+} s 0}\left(-s^{-}+s^{+}\right)\right)}{e^{2 m \mu^{+}\left(s^{+}-s^{-}\right)}}<0 .
\end{aligned}
$$

The signs of Eqs. (A.17a) and (A.17b) follow from Jensen's inequality, which states that if $U$ is convex function and $X$ is any random variable, $U\{\mathbb{E}(X)\} \leq \mathbb{E}\{U(X)\}$. The inequality is strict if $U$ is strictly convex and $X$ is non-degenerate. On the other hand, by assuming for simplicity that $s_{0}=\left(s^{+}-s^{-}\right) / 2$, we can rewrite the second term in the product of the numerator in Eq. (A.17a):

$$
e^{2 m \mu^{+} s^{+}}\left(-\frac{\Delta s}{2}\right)+e^{2 m \mu^{+} s^{-}}\left(-\frac{\Delta s}{2}\right)+e^{2 m \mu^{+} s 0}(\Delta s)
$$

where $\Delta s=s^{+}-s_{0}=s_{0}-s^{-}$by definition. Collecting then $\Delta s$ :

$$
\Delta s\left(\frac{1}{2} e^{2 m \mu^{+} s^{+}}-\frac{1}{2} e^{2 m \mu^{+} s^{-}}+e^{2 m \mu^{+} s 0}\right)
$$

Since the exponents of the terms in $A .19$ are functions of $s^{+}, s^{-}$and $s_{0}$ respectively, we can rewrite it in the following form:

$$
\Delta s\left(\frac{1}{2} e^{f\left(s^{+}\right)}-\frac{1}{2} e^{f\left(s^{-}\right)}+e^{f(s 0)}\right)
$$


a

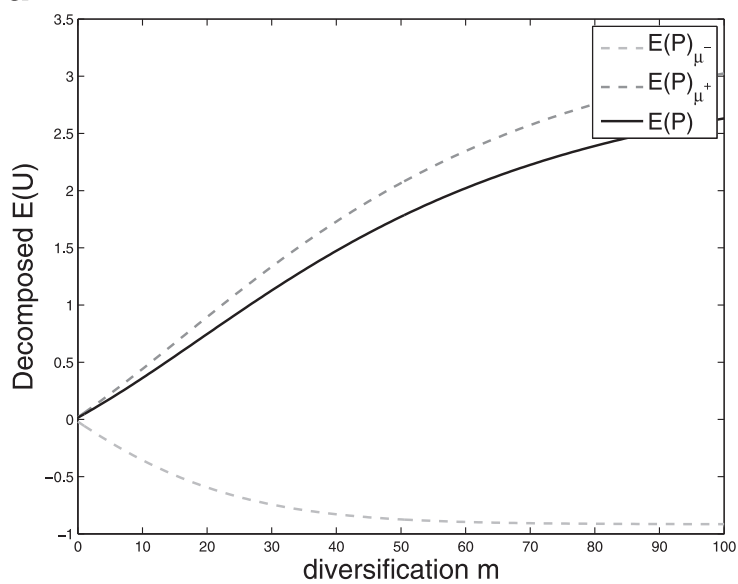

b

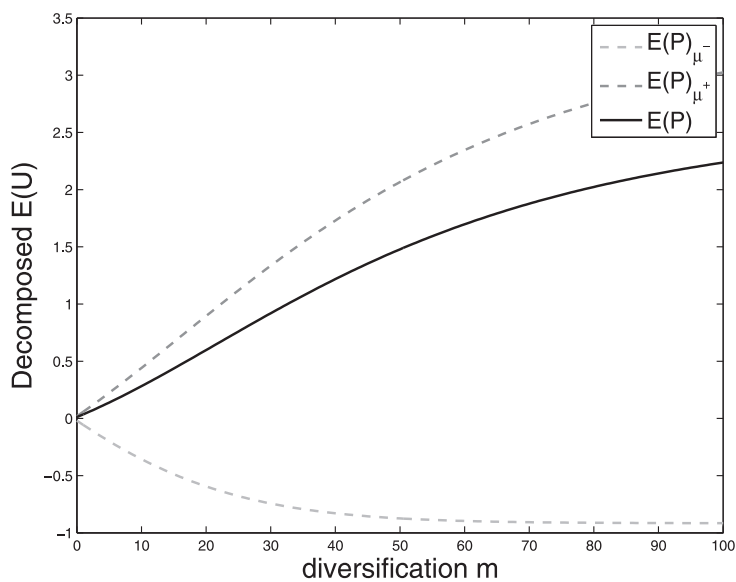

Fig. A.10. Conditional expected utility (low values of $p$ ). Conditional expected utility. Parameter: (a) $p=0.1$, (b) $p=0.2$. Parameters: $\sigma^{2}=0.5, r_{f}=0.001$, $\varepsilon=0.1, \lambda=0.1, \beta=0.2, l=0.5, m \in\{1, \ldots, 100\}, s_{0}=1, w=1,\left|\mu^{+}\right|=\mid \mu^{-}=0.003$.

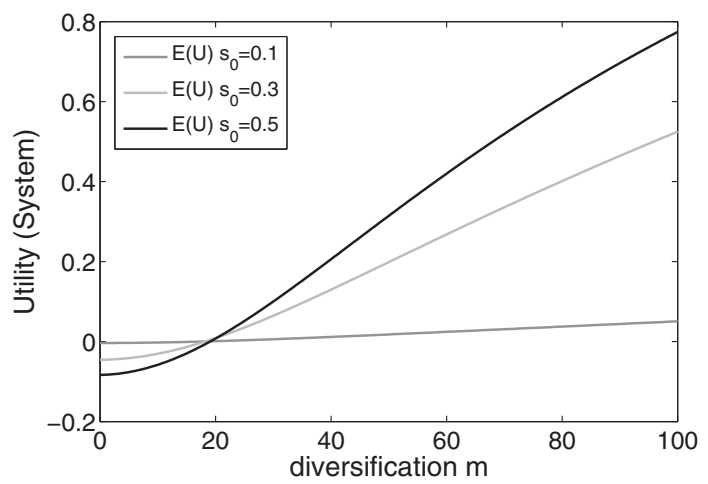

Fig. A.11. Conditional expected utility (equally likely scenarios). Comparison between expected utility functions for different values of $s_{0}$. Parameters: $s_{0} \in$ $\{0.1,0.3,0.5\}, l=0.5, p \rightarrow 0.5, \beta=0.2, m \in\{1,2, \ldots, 100\}, \epsilon=0.1, w=1, r_{f}=0.001, \lambda=0.1,\left|\mu^{+}\right|=\left|\mu^{-}\right|=0.003$.

Notice that the above function is convex, because linear combination of convex functions. From Jensen's Inequality therefore:

$$
\frac{f\left(s^{+}\right)+f\left(s^{-}\right)}{2}>f\left(\frac{s^{+}+s^{-}}{2}\right)
$$

The last result implies that the sign of the numerator of Eq. (A.17a) is positive, and hence the first derivative of $q$ with respect to $m$ will be also positive. Moreover, because of the symmetry between the two derivatives of $g$ and $q$, it follows that the first derivative of $g$ with respect to $m$ is negative.

The proof is complete once we show that the utility function $U$ can be maximized at the extreme points of the feasible range of $m$. Given the non-linear dependence of $\partial g / \partial m$ and $\partial q / \partial m$, we prove by existence that the statement is valid. Since $p \in[0,0.5)$, we can show that:

$$
\begin{aligned}
& p=0: \frac{\partial U\left(\Pi_{m}\right)_{\mu}}{\partial m}=-\frac{\partial g}{\partial m} \Delta \pi \geq 0 \\
& p \rightarrow 0.5: \frac{\partial U\left(\Pi_{m}\right)_{\mu}}{\partial m}=\frac{1}{2} \frac{\partial q}{\partial m} \Delta \pi-\frac{1}{2} \frac{\partial g}{\partial m} \Delta \pi=0 .
\end{aligned}
$$

As it turns out, considered the full interval of $p \in[0,0.5)$ we found that $\left(\frac{\partial U\left(\Pi_{m}\right)_{\mu}}{\partial m}\right)_{p=0}>\left(\frac{\partial U\left(\Pi_{m}\right)_{\mu}}{\partial m}\right)_{p \rightarrow 0.5}$. Therefore, bank expected utility function will be always increasing monotonically with the level of diversification.

The last results imply that for values of $p$ sufficiently small, the solution to the bank utility maximization problem, $\max _{m} U\left(\Pi_{m}\right)_{\mu}=U\left(\Pi_{m}\right)_{\mu^{+}}$, will be such that $m^{*}=M$ (see Fig. A.10). To conclude, by comparing Figs. 7 and A.11, we show, by existence, that extreme solutions can be obtained for values in the lower region of the parameter space of $s_{0}$. One could therefore conjecture the existence of a generic threshold $s^{*}$ below which the statement holds. See proof Proposition 5.II. 
Given Eq. 10, these conditions will correspond to healthy banks with low levels of leverage and with a relatively large asset size.

Proof. Proposition 5.II

Point II in Proposition 5 shows that in a (arbitrage free) complete market of assets with a stochastic trend, even in the absence of transaction costs banks can maximize their MV utility by selecting an intermediate level of diversification $m^{\star}$. To prove it, we will use the results of the previous proof and consider once again the two simplified equations of $g$ and $q$ in Eq. A.16 and derive the partial derivatives with respect to $s_{0}$. We can easily find that these are negative and positive respectively:

$$
\begin{gathered}
\frac{\partial q}{\partial s_{0}}=-\frac{2 e^{2 m \mu^{-}\left(s^{+}-s_{0}\right)} m \mu^{-}}{-1+e^{2 m \mu^{-}\left(s^{+}-s^{-}\right)}}<0 \\
\frac{\partial g}{\partial s_{0}}=-\frac{2 e^{2 m \mu^{+}\left(s^{+}-s_{0}\right)} m \mu^{+}}{-1+e^{2 m \mu^{+}\left(s^{+}-s^{-}\right)}}>0 .
\end{gathered}
$$

Now, we decompose the MV utility in Eq. (24) as follows:

$$
\begin{aligned}
& \mathbb{E} U\left(\Pi_{m}\right)_{\mu^{-}}=p\left[\left(q \pi^{-}+(1-q) \pi^{+}\right)-\frac{\lambda}{2}\left(q\left(\Delta_{-}^{-}\right)^{2}+(1-q)\left(\Delta_{-}^{+}\right)^{2}\right)\right], \\
& \mathbb{E} U\left(\Pi_{m}\right)_{\mu^{+}}=(1-p)\left[\left(g \pi^{-}+(1-g) \pi^{+}\right)-\frac{\lambda}{2}\left(g\left(\Delta_{+}^{-}\right)^{2}+(1-g)\left(\Delta_{+}^{+}\right)^{2}\right)\right] \\
& \text { where }\left\{\begin{array}{l}
\pi^{+}:=s^{+}-s_{0}, \\
\pi^{-}:=s^{-}-s_{0},
\end{array}\right. \\
& \text { and }\left\{\begin{array}{l}
\Delta_{-}^{-}:=\pi^{-}-p\left[q \pi^{-}+(1-q) \pi^{+}\right], \\
\Delta_{-}^{+}:=\pi^{+}-p\left[q \pi^{-}+(1-q) \pi^{+}\right], \\
\Delta_{+}^{-}:=\pi^{-}-(1-p)\left[g \pi^{-}+(1-g) \pi^{+}\right], \\
\Delta_{+}^{+}:=\pi^{+}-(1-p)\left[g \pi^{-}+(1-g) \pi^{+}\right] .
\end{array}\right.
\end{aligned}
$$

Let observe that Eq. (A.23a) is decreasing in $m$, while Eq. (A.23b) is increasing in $m$. Conversely, Eq. (A.23a) is increasing in $s_{0}$ and Eq. (A.23b) is decreasing in $s_{0}$ :

$$
\begin{aligned}
& \frac{\partial \mathbb{E} U\left(\Pi_{m}\right)_{\mu^{-}}}{\partial m}=p\left(\frac{\partial q}{\partial m}\right)\left(\pi^{-}-\pi^{+}\right)<0 \\
& \frac{\partial \mathbb{E} U\left(\Pi_{m}\right)_{\mu^{+}}}{\partial m}=(1-p)\left(\frac{\partial g}{\partial m}\right)\left(\pi^{-}-\pi^{+}\right)>0 \\
& \frac{\partial \mathbb{E} U\left(\Pi_{m}\right)_{\mu^{-}}}{\partial s_{0}}=p\left(\frac{\partial q}{\partial s_{0}}\right)\left(\pi^{-}-\pi^{+}\right)>0 \\
& \frac{\partial \mathbb{E} U\left(\Pi_{m}\right)_{\mu^{+}}}{\partial s_{0}}=(1-p)\left(\frac{\partial g}{\partial s_{0}}\right)\left(\pi^{-}-\pi^{+}\right)<0
\end{aligned}
$$

Eq. (24) in Section 4.2 can be interpreted as a linear combination of Eq. (A.23a) and Eq. (A.23b) that are weighted by $p$ and $(1-p)$, respectively.

As it turns out, the optimum will be a function of both $s_{0}$ and $m$. The conditions to be verified in order to find the probability $p^{\star}$ that makes the partial derivatives equivalent are:

$$
\begin{aligned}
& \text { FOC1 }): \quad p\left(\frac{\partial q}{\partial m}\right)\left(\pi^{-}-\pi^{+}\right)=(1-p)\left(\frac{\partial g}{\partial m}\right)\left(\pi^{-}-\pi^{+}\right) \\
& \text {FOC2 }): \quad p\left(\frac{\partial q}{\partial s_{0}}\right)\left(\pi^{-}-\pi^{+}\right)=(1-p)\left(\frac{\partial g}{\partial s_{0}}\right)\left(\pi^{-}-\pi^{+}\right) .
\end{aligned}
$$

Putting into system the two equations, it must be true that:

$$
\frac{\partial q}{\partial m}=\frac{\partial q}{\partial s_{0}} \quad \text { and } \quad \frac{\partial g}{\partial m}=\frac{\partial g}{\partial s_{0}}
$$


The conditions are satisfied for all

$$
p^{\star}=1 /\left(1+\frac{\partial q}{\partial g}\right) \in \Omega_{p^{\star}} \subset \Omega_{P}:=[0,1]
$$

with $q=q\left(m^{\star}\right), g=g\left(m^{\star}\right)$. In a more general form, Eq. (A.28) can be written as $p^{\star}=f\left[g\left(m^{\star}, s^{\star}\right) ; q\left(m^{\star}, s^{\star}\right)\right]$. Since $g, q$ and $f$ are all one-to-one, for the inversion function theorem are invertible functions. Hence, for a fixed value of $p^{\star}$, must exists $m^{\star}$ and $s^{\star}$ such that simultaneously:

$$
m^{\star}=\left[\left(g^{-1} ; q^{-1}\right) \circ f^{-1}\right]\left(p^{\star}\right) \Rightarrow \exists \quad \mathbb{E} U\left(\Pi_{m^{\star}}\right) \geq \mathbb{E} U\left(\Pi_{m}\right) \quad \forall m \gtrless m^{\star} .
$$

and:

$$
s^{\star}=\left[\left(g^{-1} ; q^{-1}\right) \circ f^{-1}\right]\left(p^{\star}\right) \Rightarrow \exists \quad \mathbb{E} U\left(\Pi_{s^{\star}}\right) \geq \mathbb{E} U\left(\Pi_{s}\right) \quad \forall s \gtrless s^{\star} .
$$

The economic growth condition implies $p^{\star}<\frac{1}{2}$. From Eq. (A.28), this is equivalent to write:

$$
\begin{aligned}
& \frac{1}{2}> \frac{\frac{\partial g}{\partial m}}{\frac{\partial q}{\partial m}+\frac{\partial g}{\partial m}} \\
& \frac{\partial g}{\partial m}<\frac{1}{2}\left[\frac{\partial q}{\partial m}+\frac{\partial g}{\partial m}\right] \\
& \frac{\partial g}{\partial m}+\frac{\partial g}{\partial m}<\frac{\partial q}{\partial m}+\frac{\partial g}{\partial m} \frac{\partial q}{\partial m} \\
& \frac{\partial m}{\partial m}
\end{aligned}
$$

which is always true because from Eqs. (A.17a)-(A.17b) $\frac{\partial g}{\partial m}<0$ and $\frac{\partial q}{\partial m}>0$. The last result implies that to each $p^{\star} \in \Omega_{p^{\star}} \subset$ $\Omega_{p}:=\left[0, \frac{1}{2}\right)$ corresponds an optimal level of diversification $m^{\star}$ in the open ball $B\left(\frac{1+M}{2}, r\right)=\left\{m^{\star} \in \mathbb{R} \mid d\left(m^{\star}, \frac{1+M}{2}\right)<r\right\}$ with center $\frac{1+M}{2}$ and radius $r \in[0, \alpha]$ where $\alpha=f(q, g)$. Then, $\mathbb{E} U\left(\Pi_{m}\right) \leq \mathbb{E} U\left(\Pi_{m^{\star}}\right)$, for all $m \notin B\left(\frac{1+M}{2}, r\right)$.

To conclude, by comparing similarly to before Figs. 7 and A.11, we show by existence, that conditional to an initial price value above a given threshold $s^{*}$, the solution to the bank maximization problem is internal.

Proposition 7. If banks do not internalize the impact of social costs in the case of their default, the banking system will be over-diversified in external assets w.r.t. to the level of diversification that is socially desirable:

$$
m^{\star} \geq m^{r}
$$

and

$$
m^{r}<\infty
$$

$\forall s_{0} \in\left(s^{-}, s^{+}\right)$.

Proof. Following the same line of reasoning used in the previous proof, we decompose $\mathbb{E} U_{r}\left(\Pi_{m}\right)$ as follows:

$$
\begin{aligned}
& \mathbb{E} U_{r}\left(\Pi_{m}\right)_{\mu^{-}}=p\left[\left(q k \pi^{-}+(1-q) \pi^{+}\right)-\frac{\lambda}{2}\left(q\left(k \Delta_{-}^{-}\right)^{2}+(1-q)\left(\Delta_{-}^{+}\right)^{2}\right)\right] \\
& \mathbb{E} U_{r}\left(\Pi_{m}\right)_{\mu^{+}}=(1-p)\left[\left(g \pi^{-}+(1-g) \pi^{+}\right)-\frac{\lambda}{2}\left(g\left(\Delta_{+}^{-}\right)^{2}+(1-g)\left(\Delta_{+}^{+}\right)^{2}\right)\right] .
\end{aligned}
$$

Eq. (A.31b) is not affected by Assumption 1 in Section 4 and remains equivalent to Eq. (A.23b). Hence, the partial derivative with respect to $m$ of Eq. (A.31b) is equal to Eq. (A.24b):

$$
\frac{\partial \mathbb{E} U_{r}\left(\Pi_{m}\right)_{\mu^{+}}}{\partial m} \equiv \frac{\partial \mathbb{E} U\left(\Pi_{m}\right)_{\mu^{+}}}{\partial m}=(1-p)\left(\frac{\partial g}{\partial m}\right)\left(\pi^{-}-\pi^{+}\right) .
$$

However, because of the factor $k$, the partial derivative with respect to $m$ of Eq. (A.31a) is steeper than Eq. (A.24a). It is easy to see that for any $k>1$,

$$
\frac{\partial \mathbb{E} U_{r}\left(\Pi_{m}\right)_{\mu^{-}}}{\partial m}=p\left(\frac{\partial q}{\partial m}\right)\left(k \pi^{-}-\pi^{+}\right)<\frac{\partial \mathbb{E} U\left(\Pi_{m}\right)_{\mu^{-}}}{\partial m}=p\left(\frac{\partial q}{\partial m}\right)\left(\pi^{-}-\pi^{+}\right)<0
$$

The condition to be verified is to find the probability $p^{r}$ that makes the two equations to be equivalent:

FOC : $\quad(1-p)\left(\frac{\partial g}{\partial m}\right)\left(\pi^{-}-\pi^{+}\right)=p\left(\frac{\partial q}{\partial m}\right)\left(k \pi^{-}-\pi^{+}\right)$. 
Discarding the trivial solution $\mu_{s}=0$, the condition is satisfied for all

$$
p^{r}=1 /\left(1+\left(\frac{\partial q}{\partial g}\right) v\right) \in \Omega_{P r} \subset \Omega_{P}:=[0,1]
$$

with $q=q\left(m^{r}\right), g=g\left(m^{r}\right)$ and $v=\frac{\left(k \pi^{-}-\pi^{+}\right)}{\left(\pi^{-}-\pi^{+}\right)}$.

In a more general form, Eq. (A.32) can be written as

$$
p^{r}=f\left(v\left[g\left(m^{\star}\right) ; q\left(m^{\star}\right)\right]\right) .
$$

Since $g, q, v$ and $f$ are all one-to-one and hence invertible functions, for a fixed value of $p^{r}$, must exists an $m^{r}$ such that

$$
m^{r}=\left[\left(g^{-1} ; q^{-1}\right) \circ v^{-} \circ f^{-1}\right]\left(p^{r}\right) .
$$

Eq. (A.32) is a decreasing function with respect to $v$ which is a constant function bigger than one because $k>1$. Then, $p^{r}$ $<p^{\star}$. This implies that the diversification level $m^{r}$ is lower than the level $m^{\star}$ :

$$
m^{r}=\left[\left(g^{-1} ; q^{-1}\right) \circ v^{-1} \circ f^{-1}\right]\left(p^{r}\right)<m^{\star}=\left[\left(g^{-1} ; q^{-1}\right) \circ f^{-1}\right]\left(p^{\star}\right)
$$

From the proof of point II of Proposition 5 it follows directly that in case of uptrend and in presence of social costs, if for the individual bank is optimal to pursue maximal level of diversification, in general it is true that $m^{r}<\infty$, as the costs in the social optimum in case of systemic default will be of too great magnitude.

Proposition 6. Denote the probability of a systemic event conditional to the default of bank $j$ by $\mathbb{P}\left(\phi \geq 1 \mid \phi_{j} \geq 1\right)$. For any value of interbank exposure $1 \geq w \geq 0$ and some $j \in\{1, \ldots, N\}$, it holds:

$$
\begin{aligned}
& \text { If } m=1: \mathbb{P}\left(\phi \geq 1 \mid \phi_{j} \geq 1\right) \rightarrow 0 \text { for } n \rightarrow \infty \\
& \text { If } m=M: \mathbb{P}\left(\phi \geq 1 \mid \phi_{j} \geq 1\right)=1 \text { for } n \rightarrow \infty .
\end{aligned}
$$

Proof. In the case $m=1$ and $1 \geq w \geq 0$, for $n \rightarrow$ inf the portfolio of banks will be independent from each other, while the exposure to the interbank equals $\frac{w}{n}$, which tends to zero for large $n$. It follows that probability of several banks to default at the same time goes to zero because their shocks are independent and the exposure of a bank to any single counterparty tends to zero. Therefore, the systemic probability of default tends to zero.

In the case $1 \geq w \geq 0$ and $m=M$, banks will suffer exactly the same shock. Because of the assumption of homogeneity in the balance sheet structure of banks, if one bank defaults also all the other banks in the system must default by definition, regardless of the interbank exposure.

\section{Appendix B. Validity of the default probability definition}

The concept of default probability based on the first passage time models is widely used in credit risk models. In our framework, we extend the standard default probability definition adopted in first passage time models where a firm goes in default when its value $a$ touches a lower default boundary $l$. $l$, in general, represents the book values of the firm's debts. Instead of using the value of the firm, as driving variable, we use the leverage, defined as: $\phi=l / a$. This is an index bounded between 0 and 1. For this reason, we need to take into account not only the default boundary 1 but also the safe boundary 0 which naturally emerges because of the change of variable. The safe boundary represents a status of "permanent" strength as vice versa, the default boundary represents a status of "permanent" stress from which one firm cannot recover. Therefore, as we will argue here below, our change of variable does not deviate our notion of default probability from the standard one.

A bank could in principle reach at time $t^{i}>0$ the safe boundary $\varepsilon$ and then only later at time $t^{i i}>t^{i}>0$ reach the default boundary at 1 . Notice, however, that the probability of such an event is very low and this may occur only in a scholastic example when: (1) the variance $\sigma$ is much larger than the trend $\mu$; (2) the two barriers $s^{+}-s^{-}$are very closed to each other; and (3) the initial value of external assets $s_{0}$ is very close to the boundaries.

To test the conditions mentioned above, we performed a simple numerical test. In Fig. B.12, we plot the utility function of a bank by using fixed values of $\pi^{-}$and $\pi^{+}$. In so doing, we eliminate the dependence of the expected payoffs, in the two scenarios considered in the model, from the distance of $s_{0}$ from the upper safe boundary. We test both for the case in which maximal losses $\pi^{-}$is larger than maximal profits $\pi^{+}$and for the symmetric case.

The results confirm that the presence of an additional safe boundary in our framework does not have a significant impact on the optimal diversification strategy of banks in the mean-variance utility maximization problem.

\section{Appendix C. Monte Carlo simulations}

In order to investigate how the conditional probability of systemic default varies with the diversification $m$, for a given density of the interbank network, we perform a set of Monte Carlo simulations. ${ }^{16}$ In a nutshell:

\footnotetext{
16 The simulation procedure is described in more detail in C.1.
} 
a

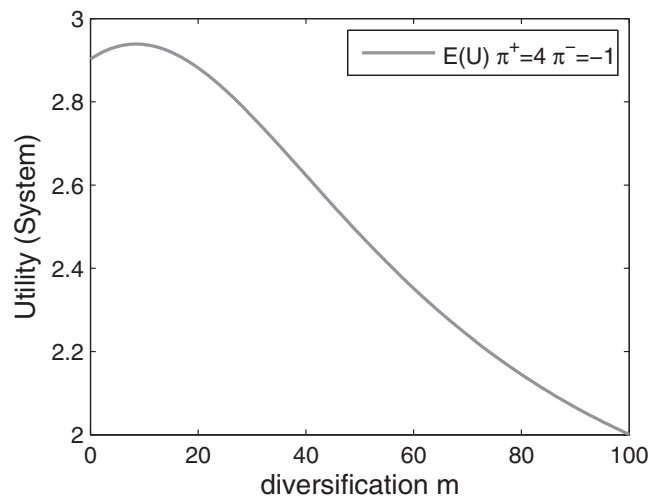

b

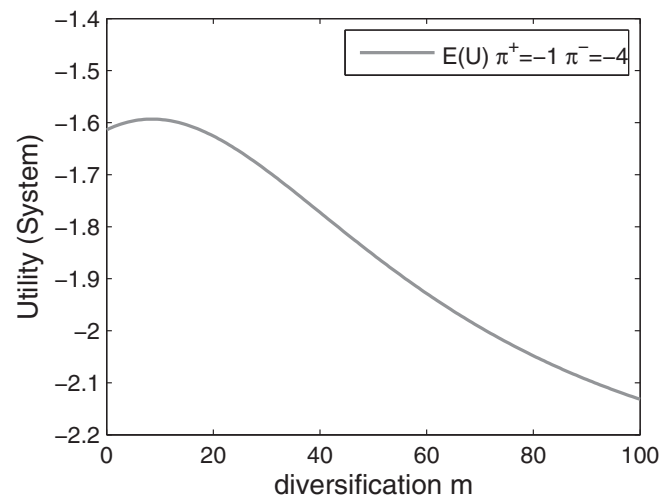

Fig. B.12. Expected utility fixing maximal losses and maximal profits. Comparison of the utility function eliminating the dependence of the payoff structure from the upper and lower barrier. (a) $\pi^{+}=1, \pi^{-}=-1$; (b) $\pi^{+}=1, \pi^{-}=-4$. Parameters: $\sigma^{2}=0.5, l=0.5, s_{0}=3.7, p=.4, \beta=0.2, r_{f}=0.001, \epsilon=0.1$, $m \in\{1, \ldots, 100\}$.

- We assume that, at every time step, each external asset is subject to an idiosyncratic shock. Because banks hold portfolios of such assets, their portfolio are subject to the corresponding combination of shocks.

- As diversification of banks increases, the overlap between portfolios also increases and so does the similarity of the shocks hitting banks' portfolios.

- When diversification is maximal, i.e., $m=M$, banks' portfolios are identical and are hit simultaneously by the same vector of shocks. In this extreme case, conditional to the default one bank, the probability of a systemic default is simply equal to one.

Unfortunately, because of correlation effects, there is no closed-form expression to describe mathematically the conditional systemic default probability (Frey and McNeil, 2003). Therefore, in our simulation framework we consider a system composed of $\mathrm{N}(=25)$ banks (with homogeneous balance sheet structure but heterogeneous portfolios of external assets) and approximate the conditional systemic default probability by the frequency of simultaneous defaults occurring after the default of the first bank. In practice, given the default of the first bank at time $t_{p}$, we test and measure the frequency of defaults among the $N-1$ banks during two alternative time windows: 5 time steps $t_{p+1}, \ldots, t_{p+5}$ and 10 time steps $t_{p+1}, \ldots, t_{p+5}, \ldots, t_{p+10}$.

In order to perform our tests, we first simulate the Brownian motion in the scenario of a negative trend and compare the values of leverage, among the $N$ banks, given a stochastic value of $w$ and with other parameters (e.g., $\left.l, s_{0}\right)$ kept fixed. Under the assumption of homogeneity in the balance sheet structure of the banks, the leverage price-cycle used in the Monte Carlo analysis is specified as follows:

$$
\left\{\begin{array}{l}
\frac{d s_{i}}{s_{i}(t)}=\mu d t+\frac{\sigma}{\sqrt{m}} d B_{p} \\
\phi_{i}=\frac{l_{i}}{s_{i}+w_{i} \frac{l_{i}}{1+r_{f}+\beta \phi_{j}}}
\end{array}\right.
$$

The trajectories of the leverages in two selected runs of the simulations are shown in Fig. C.13. Grey lines represent the leverage of each bank over time and the blue lines represent the average of the leverage values across $(N-1)$ banks.

For a sample of 1000 runs, Fig. C.14a and b show respectively the average time to default and the number of total defaults occurred in each run of the simulation at different diversification levels, $m \in\{1,25,50,75,100\}$. As expected, diversification brings immediate benefits even at low levels. Since we are interested in the conditional systemic default event, we count the number of simultaneous default. Namely, the number of banks that simultaneously collapse within a short time window, after an initial individual default. Specifically, the time window is set equal to 5 time steps in Fig. C.15a and to 10 time steps in Fig. C.15b. For a number $\mathrm{M}(=100)$ of external assets, Fig. C.15 shows that the number of simultaneous defaults increases with the level of portfolio diversification. In particular, if all the banks were fully diversified, i.e., if $\mathrm{m}=\mathrm{M}$, all the $\mathrm{N}$ banks would default simultaneously.

\section{C1. Simulation procedure}

To verify numerically the Proposition 6, we study the dynamics of $\phi$ and the number of simultaneous defaults among the $\mathrm{N}-1$ banks after the first bank goes in default. 
a

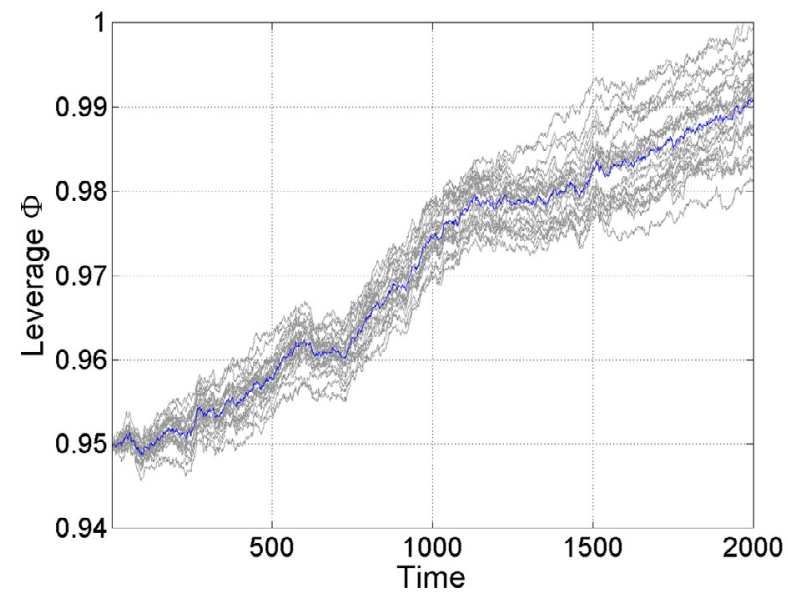

b

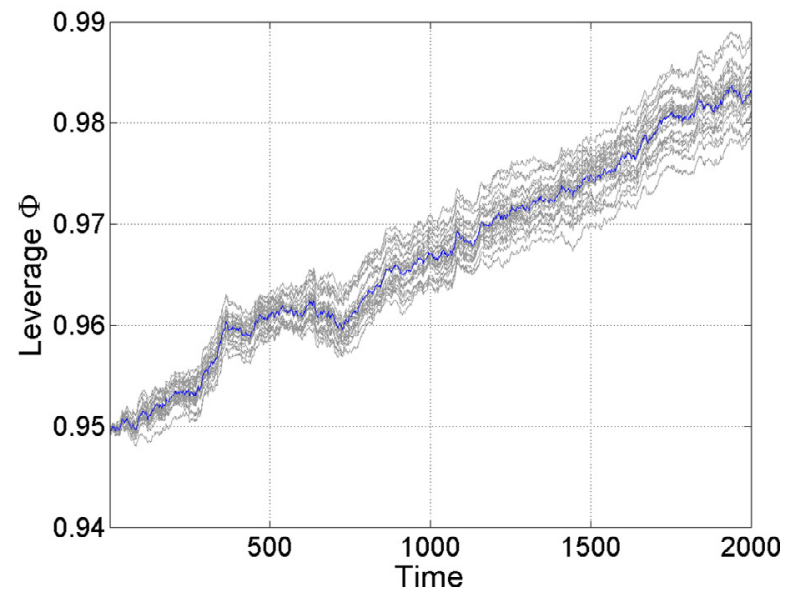

Fig. C.13. Statistics about $\phi$ trajectories and banks' exit time. Trajectories and exit time obtained for $n$ banks (grey lines) and their average (blue line) from the system of Eq. C.1. Parameters chosen for the simulation $\phi=0.95$, number of banks $N=25, \beta=0.7, l=.5, \mu=-0.04, w=0.5$. (a) Trajectories of banks $\phi$ for $m=50$; (b) Trajectories of banks $\phi$ for $m=75$. (For interpretation of the references to color in this figure legend, the reader is referred to the web version of this article.)

a

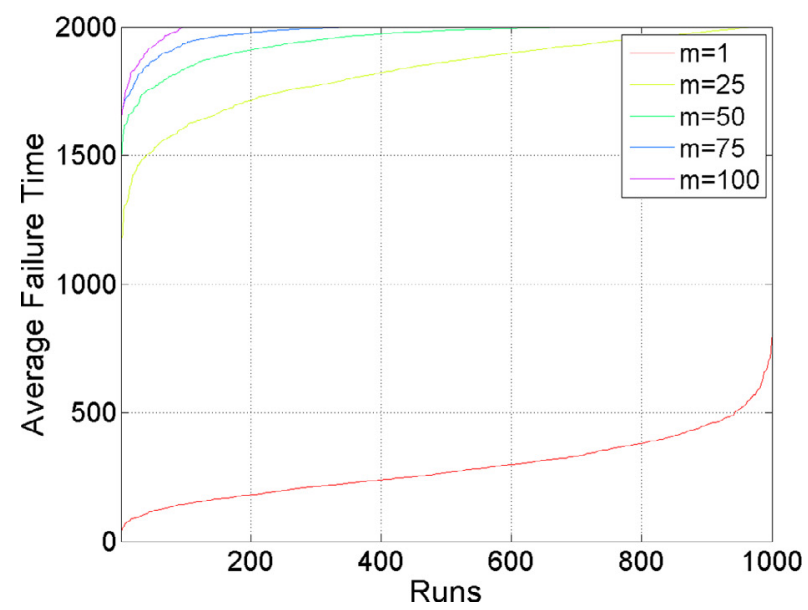

b

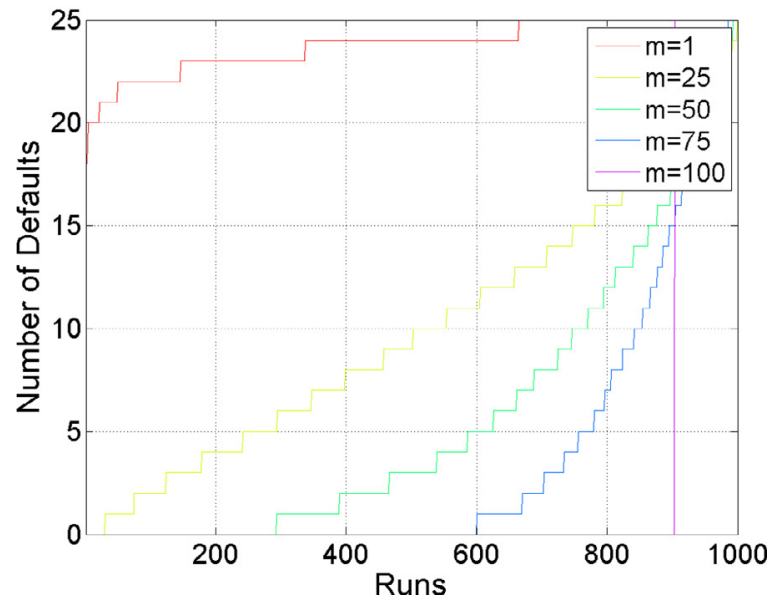

Fig. C.14. Statistics on banks simultaneous defaults. Parameters are in both panels as in Fig. C.13. (a) Average failure time for $m \in\{1,25,50,75,100\}$; (b) Number of total defaults across banks for $m \in\{1,25,50,75,100\}$.

Consider in particular to run many times a stochastic process describing the leverage of the banks in the system with an upper barrier at one and to restart it from the same initial condition $\phi_{0}<1$. We stop the process once all the banks exit this barrier.

Furthermore, we consider a discretized version of Brownian motion, we set the step-size as $\Delta t$ and let $B_{p}$ denote $B\left(t_{p}\right)$ with $t_{p}=p \Delta t$. According to the properties of Brownian motion, we find:

$$
B_{p}=B_{p-1}+d B_{j} \quad p=1, \ldots, P
$$

where $P$ denotes the number of steps that we take with $t_{0}, t_{1}, \ldots, t_{P}$ as a discretization of the interval $[0, T]$, and $d B_{p}$ is a vector of normally distributed random variables $k x 1$ with zero mean and variance $\Delta_{t}$. In our case $k$ corresponds to the number of external assets held by the individual bank. Computing numerically Eq. (15) we can simulate a Brownian motion on the external assets prices.

To simulate the stochastic dynamical system of equations, we follow the simple Euler discretization scheme. We first discretize the dynamics of the processes $s_{i, t \geq 0}$. Then, we substitute the value of $s$ and $l$ into the discrete time version of the last expression into the above system of equations. It is unnecessary to derive the dynamics of $\phi_{i, t} \geq 0$ via Itos Lemma 
a

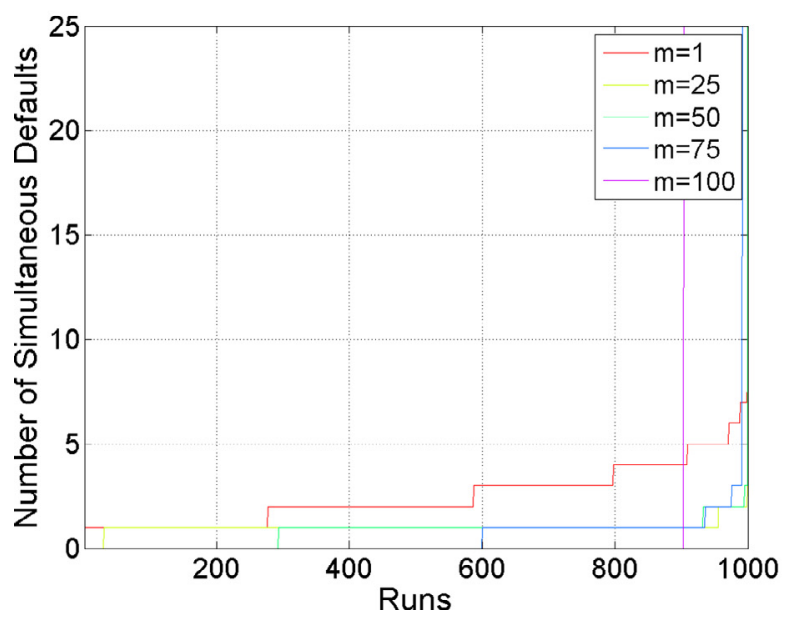

b

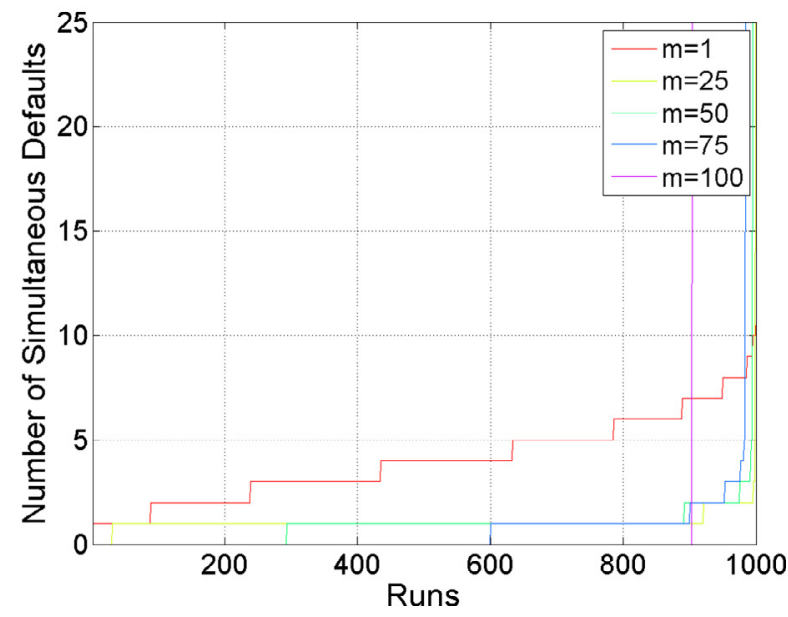

Fig. C.15. Statistics on simultaneous defaults. After one bank defaults, we keep track of the number of banks occurred within a certain time window. Parameters are set in both panels as in Fig. C.13. (a) Simultaneous defaults within 5 time steps; (b) Simultaneous defaults within 10 time steps.

from the dynamics of $s_{i, t \geq 0}$. The resulting discrete version of the system of equations above is:

$$
\left\{\begin{array}{l}
s_{i, p}=s_{i, p-1}+\mu_{s}\left(\phi_{i, p-1}, s_{p-1}, \phi\right) \Delta t+\sigma\left(s_{i, p-1}\right) d B_{p} \\
\phi_{i, p}=\frac{l_{i, p}}{s_{i, p}+w_{i, p} \frac{l_{i, p}}{1+r_{f}+\beta \phi_{j, p}}}
\end{array}\right.
$$

where $\phi_{i, p}$ and $s_{i, p}$ are the approximations to $\phi_{i}(p \Delta t)$ and $s_{i}(p \Delta t)$, while $\Delta t$ is the step size, $d B_{p}=B_{p}-B_{p-1}$ and $P$ is the number of steps we take in the process.

We run Monte Carlo simulations for a total of $N$ sample paths within [0,T], i.e. economy terminal date. From $t=1$, we measure the time step $T$ at which the process $\left\{\phi_{i}\right\}_{t>0}$ crossed the default boundary fixed at one for each bank $i$. We stop the process when all the banks in the system reached this point and keep track of the fraction of trajectories that crossed the default boundary after the default of one bank in a given number of time steps. We make sure that first bank defaults at time $T$ are not accounted for.

\section{References}

Atkeson, A.G., Eisfeldt, A.L., Weill, P.-O., 2013. Measuring the Financial Soundness of US Firms 1926-2012. Federal Reserve Bank of Minneapolis Research Department Staff, p. 484. Report

Battiston, S., Delli Gatti, D., Gallegati, M., Greenwald, B.C.N., Stiglitz, J.E., 2012a. Credit default cascades: when does risk diversification increase stability? J. Financial Stab. 8 (3), 138-149.

Battiston, S., Gatti, D.D., Gallegati, M., Greenwald, B.C.N., Stiglitz, J.E., 2012b. Liaisons dangereuses: increasing connectivity, risk sharing, and systemic risk. J. Econ. Dyn. Control 36 (8), 1121-1141.

Battiston, S., Puliga, M., Kaushik, R., Tasca, P., Caldarelli, G., 2012c. Debtrank: Too central to fail? Financial networks, the fed and systemic risk. Sci. Rep. 2. 541.

Billingsley, P., 1968. Convergence of Probability Measures. John Wiley \& Sons.

Bird, R., Tippett, M., 1986. Note-Naive Diversification and Portfolio Risk-A Note.. Manag. Sci. 32 (2), $244-251$.

Black, F., Cox, J., 1976. Valuing corporate securities: some effects of bond indenture provisions. J. Finance 31 (2), $351-367$.

Brock, W., Hommes, C., Wagener, F., 2009. More hedging instruments may destabilize markets. J. Econ. Dyn. Control 33 (11), 1912-1928.

Cifuentes, R., Ferrucci, G., Shin, H.S., 2005. Liquidity risk and contagion. J. Eur. Econ. Assoc. 3 (2-3), 556-566.

Collin-Dufresne, P., Goldstein, R.S., Martin, J.S., 2001. The determinants of credit spread changes. J. Finance 56 (6), $2177-2207$.

Danielsson, J., Zigrand, J.-P., 2008. Equilibrium asset pricing with systemic risk. Econ. Theory 35 (2), $293-319$.

Eisenberg, L., Noe, T., 2001. Systemic risk in financial systems. Manag. Sci. 47 (2), 236-249.

Elsinger, H., Lehar, A., Summer, M., 2006. Risk assessment for banking systems. Manag. Sci. 52 (9), $1301-1314$.

Elton, E.J., Gruber, M.J., 1977. Risk reduction and portfolio size: an analytical solution. J. Bus. 50 (4), $415-437$.

Evans, J., Archer, S., 1968. Diversification and the reduction of dispersion: an empirical analysis. J. Finance 23 (5), $761-767$.

Frey, R., McNeil, A., 2003. Dependent defaults in models of portfolio credit risk. J. Risk 6, 59-92.

Fricke, D., Lux, T., 2015. Core-periphery structure in the overnight money market: evidence from the e-mid trading platform. Comput. Econ. 45 (3), 359-395.

Gardiner, C.W., 1985. Handbook of Stochastic Methods for Physics, Chemistry, and the Natural Sciences. Springer.

Goldstein, I., Pauzner, A., 2004. Contagion of self-fulfilling financial crises due to diversification of investment portfolios. J. Econ. Theory 119 (1), 151-183.

Haldane, A., 2009. Rethinking the financial network. In: Speech delivered at the Financial Student Association, Amsterdam.

Ibragimov, R., Jaffee, D., Walden, J., 2011. Diversification disasters. J. Financial Econ. 99 (2), 333-348.

Iori, G., Jafarey, S., Padilla, F., 2006. Systemic risk on the interbank market. J. Econ. Behav. Org. 61 (4), 525-542.

Johnson, K., Shannon, D., 1974. A note on diversification and the reduction of dispersion. J. Financial Econ. 1 (4), $365-372$.

Maillard, S., Roncalli, T., Teiletche, J., 2010. The properties of equally weighted risk contribution portfolios. J. Portf. Manag. 36, 1-11.

Mainik, G., Embrechts, P., 2012. Diversification in heavy-tailed portfolios: properties and pitfalls. In: Annals Actuarial Science. 
Markowitz, H., 1952. Portfolio selection. J. Finance 7 (1), 77-91.

Merton, R., 1974. On the pricing of corporate debt: the risk structure of interest rates. J. Finance 29 (2), 449-470.

Rothschild, M., Stiglitz, J.E., 1971. Increasing risk ii: its economic consequences. J. Econ. Theory 3 (1), 66-84.

Roukny, T., Bersini, H., Pirotte, H., Caldarelli, G., Battiston, S., 2013. Default cascades in complex networks: Topology and systemic risk. Sci. Rep. 3, 2759.

Samuelson, P.A., 1967. General proof that diversification pays. J. Financial Quant. Anal. 2 (1), 1-13.

Shin, H., 2008. Risk and liquidity in a system context. J. Financial Intermed. 17, 315-329.

Statman, M., 1987. How many stocks make a diversified portfolio? J. Financial Quant. Anal. 22 (03), 353-363.

Stiglitz, J., 2010. Risk and global economic architecture: why full financial integration may be undesirable. Am. Econ. Rev. 100 (2), $388-392$.

Tasca, P., 2013. Overlapping Correlation Coefficient. ETH Risk Center-Working Paper Series. ETH-RC-13-004.

Tasca, P., Mavrodiev, P., Schweitzer, F., 2014. Quantifying the impact of leveraging and diversification on systemic risk. J. Financial Stab. 15, 43-52.

Tobin, J., 1958. Liquidity preference as behavior towards risk. Rev. Econ. Stud. 25 (2), 66-86.

Wagner, W., 2010. Diversification at financial institutions and systemic crises. J. Financial Intermed 19 (3), 373-386.

Wagner, W., 2011. Systemic liquidation risk and the diversity diversification tradeoff. J. Finance 66 (4), 1141-1175.

Windcliff, H., Boyle, P., 2004. The 1/n pension investment puzzle. North Am. Actuar. J. 8, 32-45.

Zhou, C., 2010. Dependence structure of risk factors and diversification effects. Insur. Math. Econ. 46 (3), 531-540. 